This is the final peer-reviewed accepted manuscript of:

Alberto Martini, Marco Troncossi, Alessandro Rivola

Algorithm for the static balancing of serial and parallel mechanisms combining counterweights and springs: Generation, assessment and ranking of effective design variants

In: Mechanism and Machine Theory, Volume 137, 2019, Pages 336-354

The final published version is available online at:

https://doi.org/10.1016/j.mechmachtheory.2019.03.031

Rights / License:

The terms and conditions for the reuse of this version of the manuscript are specified in the publishing policy. For all terms of use and more information see the publisher's website.

This item was downloaded from IRIS Università di Bologna (https://cris.unibo.it/)

When citing, please refer to the published version. 


\title{
Algorithm for the Static Balancing of Serial and Parallel Mechanisms Combining Counterweights and Springs: Generation, Assessment and Ranking of Effective Design Variants
}

\author{
Alberto Martini ${ }^{\mathrm{a},{ }^{*},}$, Marco Troncossi ${ }^{\mathrm{a}}$, Alessandro Rivola ${ }^{\mathrm{a}}$ \\ ${ }^{a}$ DIN - Dept. of Engineering for Industry, University of Bologna, \\ V.le del Risorgimento 2 - 40136 Bologna, Italy \\ *Corresponding author, e-mail: alberto.martini6@unibo.it
}

https://doi.org/10.1016/i.mechmachtheory.2019.03.031

(c) 2019. This manuscript version is made available under the CC-BY-NC-ND 4.0 license http://creativecommons.org/licenses/by-nc-nd/4.0/

\begin{abstract}
Static balancing through passive devices is a suitable strategy to reduce motor loads for numerous applications in the automation and robotics fields. Many known methods require initially defining which balancing elements to install, thus possibly limiting the compensation effectiveness, since potentially optimal solutions may be neglected. This work presents an approach to statically balance linkages characterized by open and/or closed kinematic chains. The proposed algorithm searches for possible balanced variants of the mechanism that can be arranged by installing combinations of counterweights and springs, without auxiliary linkages. If solutions are found, the corresponding balancing parameters are tuned for optimizing the mechanism energy consumption, by considering the mechanism dynamics when performing its operational tasks. Actual benefits and drawbacks of the variants are assessed through quantitative criteria. The corresponding performance indicators are proposed as a guideline for designers to identify the most convenient balancing solutions. The implemented procedure is general and suitable to study any mechanism admitting closed-form solutions for its forward kinematics. A case study concerning an industrial palletizing robot is reported as an example of application. Overload issues affecting the robot actuators are solved through gravity compensation. The results achieved for the industrial problem prove the procedure effectiveness.
\end{abstract}

Keywords: static balancing, gravity compensation, serial manipulator, parallel robot, palletizing robot.

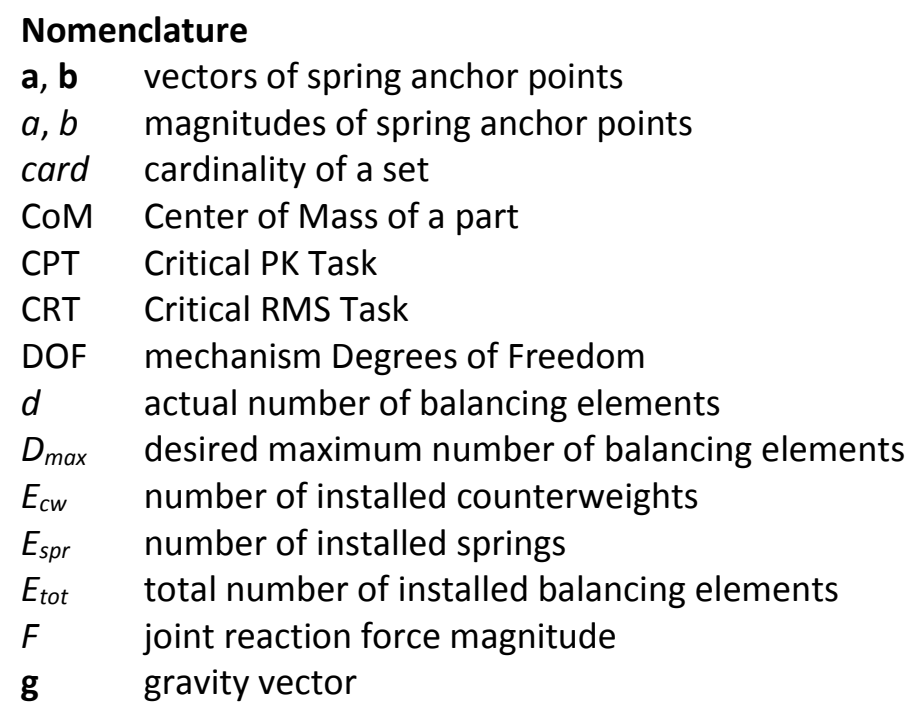




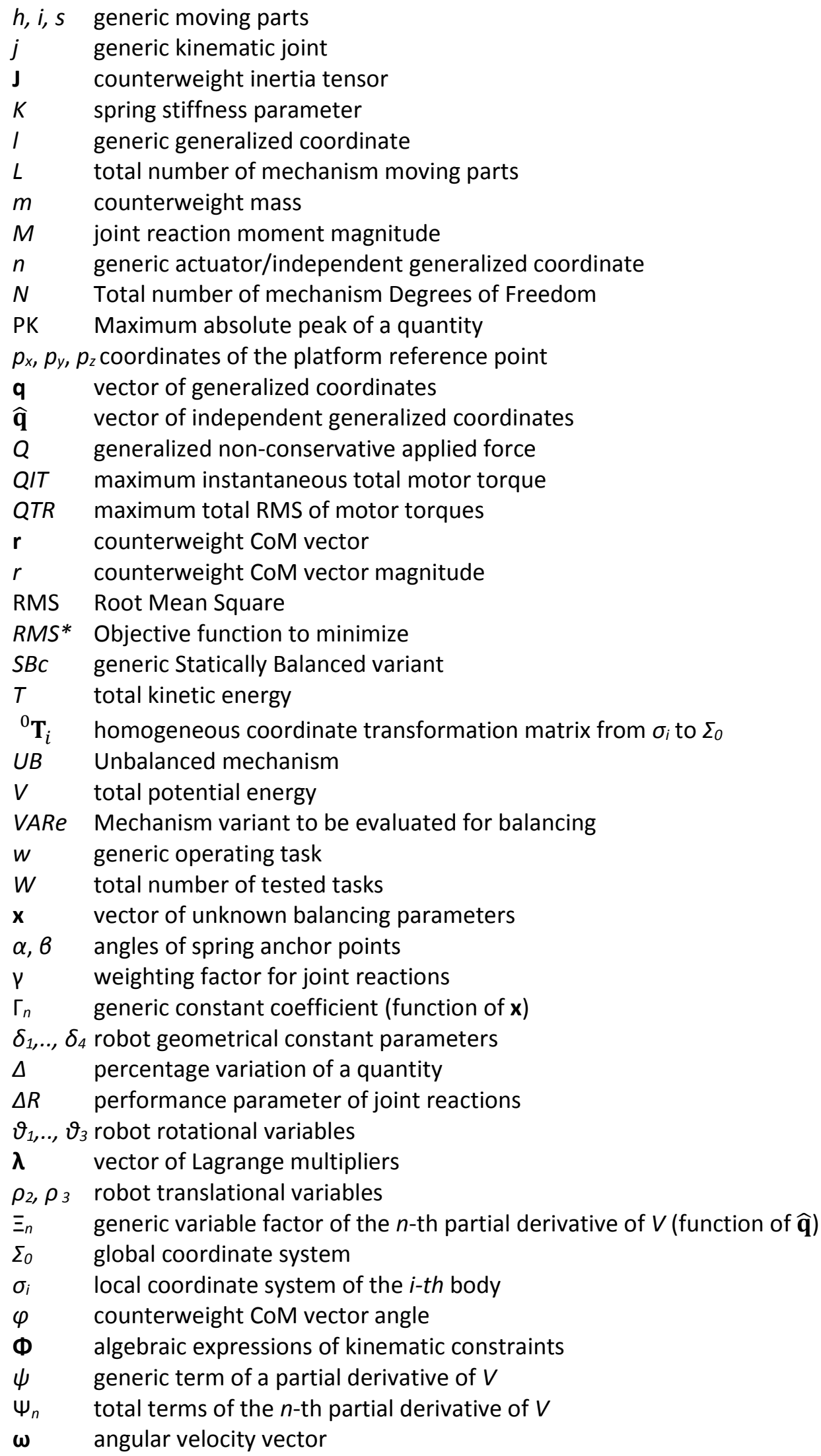

\section{Introduction}

A mechanism is statically balanced if its total potential energy is invariant for any admissible configuration. Under this condition, the mechanism is constantly in neutral equilibrium and its actuators are not required to sustain any conservative force acting on its moving members. In case static balancing is only adopted for 
balancing the weight of the mechanism moving members (i.e. the gravitational forces), which is the most common application, the mechanism can be also referred to as "gravity compensated". Several approaches have been proposed for achieving gravity compensation [1-11]. In general, they are based on the installation of passive balancing devices (i.e. not actuated), typically counterweights and/or springs. Auxiliary linkages for properly appending such elements are frequently adopted as well [12-15].

Static balancing permits to decrease the motor actions if the gravity loads are not negligible with respect to the inertia actions. It is an essential strategy for serial manipulators commonly adopted in industrial applications [16-19] and is proven convenient also for parallel robots operating at low/moderate dynamics $[8,12,14,20]$.

The determination of effective balancing solutions for mechanisms not yet investigated in the literature may result a rather demanding goal. Firstly, most of the known balancing methods focus either on a very specific kinematic architecture or on a family of mechanisms, a general formulation still being unavailable [14, 20-22]. Secondly, a priori assumptions on the type and the location of the balancing elements to be attached are generally required $[1,5,8]$. These initial assumptions, that disregard the study of possible solutions, may prevent potentially suitable balanced variants from being identified, hence limiting the benefits possibly achievable. Thirdly, exact solutions to the balancing problem may not exist at all. Furthermore, in case feasible solutions are found, their effects on the mechanism operation must be properly assessed in order to verify their actual benefits and drawbacks [14, 23-25]. This is particularly true if multiple variants characterized by completely different arrangements of the balancing elements are available. Indeed, in such an instance, identifying the most convenient balancing solution may result a challenging task. Therefore achieving gravity compensation in practice may turn into a time-consuming trial-and-error process. Nonetheless, to the authors' best knowledge, only a few studies investigated the "automated" determination of feasible balancing solutions for linkages with certain architecture, all of them being primarily focused on serial robotic arms [21, 26-28].

This study aims at developing a design tool to assist the static balancing process, thus making the practical implementation of gravity compensation strategies more straightforward. A novel procedure to determine feasible energy-efficient balancing solutions based on proper combinations of counterweights and/or springs is here presented. A detailed analysis of merits and drawbacks characterizing both kinds of balancing elements has been provided by Carricato and Gosselin in [8]. In particular, counterweights may be easier to design and implement, and grant static balancing independently from the orientation of the gravity vector; however, they can significantly increase the mass and inertia of the mechanism, hence possibly causing negative effects for its dynamic operation (e.g. higher motor loads and lower natural frequencies of the system [8]). Springs typically add little mass and inertia to the system; nonetheless, they are more prone to generate problems of interference with the other moving parts (in case the springs are required to cover long spans) and safety issues related to the installation and maintenance of mechanical energy storage devices arise too. Balanced mechanisms with counterweights and springs adopted simultaneously may represent a convenient solution for maximizing the aforementioned benefits and limiting the potential disadvantages. Indeed, hybrid balancing solutions can be found in some industrial applications (e.g. heavy-duty serial manipulators [17]). Hence, the presented procedure can handle hybrid balancing solutions as well.

The proposed procedure is suitable for open-chain, closed-chain and hybrid serial-parallel architectures that admit closed-form solutions for their forward kinematics. Unlike most of the approaches available in the literature, the proposed procedure only requires to define as input the admissible total number of balancing elements to be installed, without any assumption on their arrangement. The procedure searches for balancing solutions among the set of all the possible mechanism variants that can be arranged with the assigned number of elements, without adding auxiliary linkages. The latter assumption (i.e. not allowing auxiliary linkages) is made in order to limit the dimensions of the balancing problem, hence permitting to automate the process by neglecting some classes of possible solutions. It may be noticed that a general formulation for the static balancing problem is still lacking and, to the authors' best knowledge, the methods currently available in the literature do not permit to assess a priori the existence of balancing solutions for a generic mechanism. Indeed, the proposed algorithm does not guarantee to yield balancing solutions, like the other known methods, but can handle general mechanisms having the features described above. 
In case analytical solutions exist and are found, the parameters characterizing the balancing elements (e.g. mass and location of a counterweight) are determined through a numerical optimization algorithm, since in a static balancing problem some of the parameters can be arbitrarily imposed. The objective function is based on a rough estimate of the mechanism energy consumption for typical operating cycles. Indeed, improved energy efficiency is one of the main advantages expected from static balancing [8], as well as an increasingly stringent requirement of modern industrial machineries. Static balancing can cancel the effects of gravity on the actuators, but the dynamic loads associated with the mechanism operation are still present, and they can be even increased by the additional mass and inertia of the balancing elements, hence limiting the benefits of compensation. Therefore, the objective function is evaluated by assessing the motor loads required for performing a proper set of typical working tasks.

Finally, the dynamic performance of the original mechanism and of its balanced variants with optimized parameters is evaluated by means of three quantitative criteria, defined according to the most relevant benefits and drawbacks potentially brought about by static balancing [8]. Consistently with the optimization function, the first and most important criterion is related to the estimated energy required over a working cycle. The second criterion takes into account the maximum instantaneous motor loads, which affect the size of motors. The third one assesses one of the major potential drawbacks, namely possible increments in the joint reactions. Three simple scalar indicators - one for each criterion - are proposed to directly compare and rank all the feasible solutions generated by the balancing procedure.

The proposed approach was applied to a case study concerning an industrial 4-DOF palletizing robot. The robot actuators experienced overload issues and excessive power consumption when performing certain trajectories. The problem was addressed by implementing gravity compensation. Due to the manipulator features (in particular the presence of actuated prismatic joints and hybrid serial-parallel kinematics), this application appeared particularly interesting to test the effectiveness of the developed procedure.

The paper has the following structure. Section 2 presents the procedure developed for achieving balancing and determine the most convenient balanced variants. Section 3 shows the results obtained for the examined palletizing robot. Section 4 draws the conclusions.

\section{Identification of effective balancing solutions}

The flowchart shown in Fig. 1 summarizes the proposed procedure. The procedure consists of the following consecutive main phases.

- $\quad$ Phase 1. The working performance of the (original) unbalanced mechanism is assessed by numerically solving its inverse dynamics for a set of prescribed tasks (provided in input by the user). The mechanism operation is evaluated in terms of proper performance indicators related to energy efficiency, peak motor actions and joint reactions (listed in order of priority), and the most critical tasks according to such criteria are identified.

- Phase 2. Feasible statically balanced variants of the original mechanism are determined and refined through an optimization process, and their performance is assessed. Firstly, all the possible variants with the desired number of balancing elements are generated. For each variant, the conditions for achieving static balancing are checked by solving symbolic relations derived from the analytical expression of its total potential energy; if a solution is found, the variant can grant exact gravity compensation and a further iterative two-step process is performed.

1. The balancing parameters describing the examined variant are adjusted through numerical optimization for minimizing an objective function related to the mechanism energy requirements associated with the critical tasks. At the first iteration, such tasks coincide with the ones determined in Phase 1 for the unbalanced mechanism.

2. The operation of the examined variant is assessed through the same procedure adopted for the analysis of the unbalanced mechanism, hence determining its performance indicators and its own critical tasks. If new critical tasks are found, the objective function is updated and a new iteration is perfomed.

- Phase 3. The performance indicators of the balanced variants determined in Phase 2 are compared with the ones obtained for the unbalanced mechanism in Phase 1. The balanced variants are ranked 
from the best to the worst according to the priority of the indicators, thus permitting to determine the most convenient balancing solutions consistently with the adopted criteria.

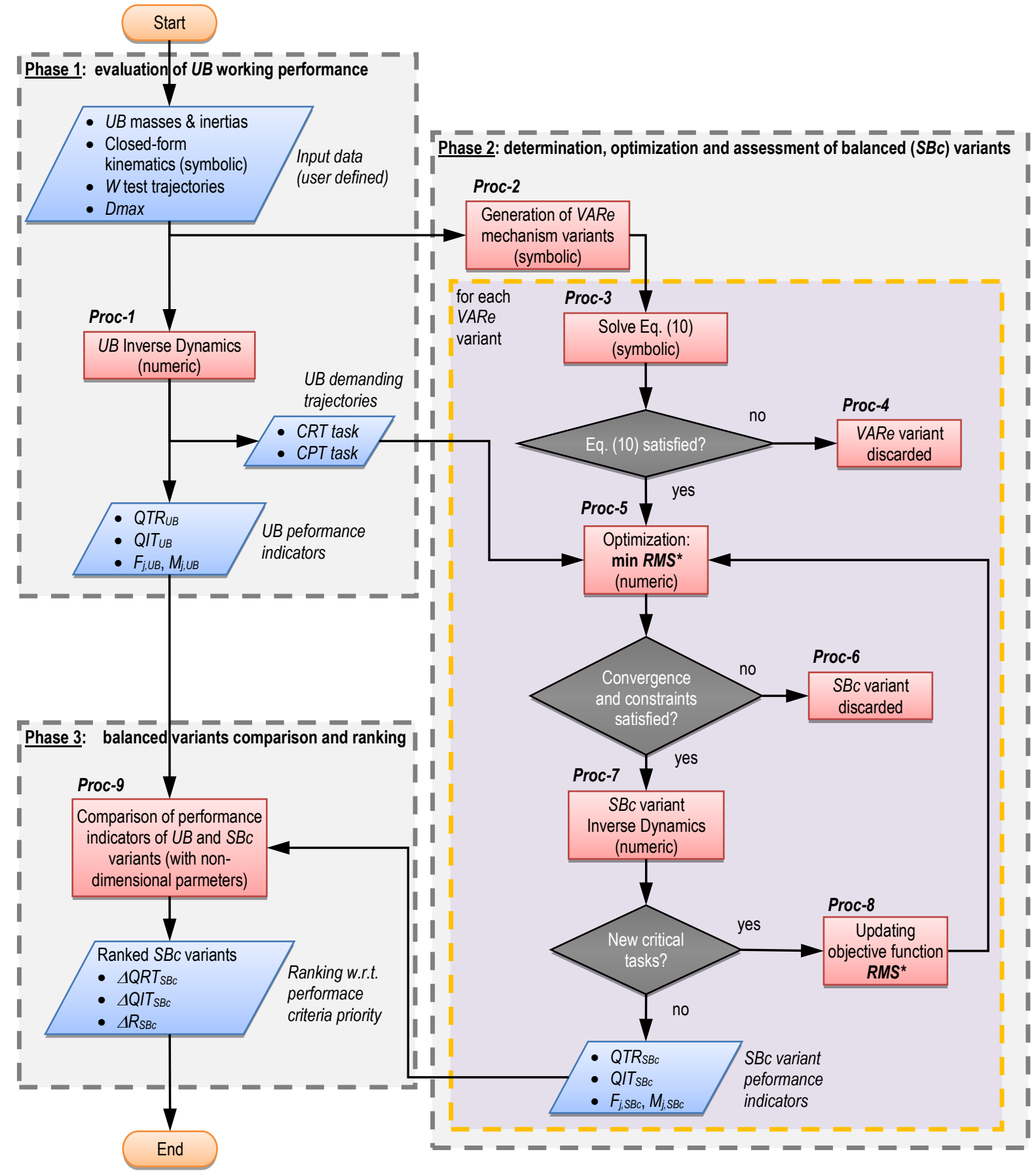

Figure 1. Flowchart of the proposed procedure.

The operating performance of the original mechanism and its balanced variants is assessed on the basis of the following three criteria, described in order of priority.

a) The first criterion is related to energy efficiency. A proper estimate of the actual electric power consumption would require detailed information on the motors and their drivers, hence lacking generality. A criterion for assessing the effectiveness of balancing in terms of mechanical work was proposed in [4] (namely, the ratio between the work of the unbalanced mechanism and the work of the balanced variant required for performing the same task). However, it does not appear suitable in case of static or quasi-static operation. Indeed, on one hand, the mechanical work is null when the motors 
are stalling; on the other hand, the unbalanced mechanism can exhibit high power consumption even in static conditions, due to the static torques (and the corresponding stall currents), whereas the balanced variants do not drain any energy. Hence, mechanical-work-based criteria are not considered satisfactory for the purpose of this study. Another criterion, conceived to be suitable also for actuated balancing systems and based on the "consumed energy", was presented in [29]; however, such work does not specify how energy can be estimated for a mechanical model. In light of the above considerations and issues, the simple trend of the motor torques over a proper set of working cycles has been considered acceptable for a rough estimate of the energy requirements (torque being proportional to the input current). Accordingly, the scalar indicator QTR is adopted, namely the Maximum Total Root Mean Square (RMS) value of the motor torques, computed as:

$$
Q T R=\max _{w}\left\{\left(\sum_{n=1}^{N} Q_{R M S, n}\right)_{w}\right\} ; w=1, . ., W
$$

where $Q_{R M S, n}$ is the torque RMS value of the $n$-th motor, considering the $w$-th operating task. Hence, QTR provides an indication on the energy requirements of the most demanding task.

b) The second criterion is related to the instantaneous motor torque, since a possible reduction in the torque peak values is one of the main benefits expected from static balancing [8]. Such a reduction may result particularly profitable in case the size of some motors can be reduced in the mechanism design. The scalar parameter QIT is adopted, namely the maximum instantaneous total motor torque, defined as:

$$
Q I T=\max _{\hat{t}, w}\left\{\left(\sum_{n=1}^{N}\left|Q_{n}(\hat{t})\right|\right)_{w}\right\} ; w=1, . ., W
$$

where $\left|Q_{n}(\hat{t})\right|$ is the magnitude of the $n$-th motor torque computed at the $\hat{t}$-th time step of simulation, related to the $w$-th operating task. It is worth noting that QIT, although not providing direct insight into the behavior of each motor (since it combines the information concerning all the motors), has been chosen for an easier comparison of the balanced variants.

c) The third criterion is related to joint reactions. Indeed, the implementation of a balancing strategy may increase their magnitude, particularly for the joints between members connected by balancing springs $[8,14,25]$. In case of remarkable overloads with respect to the original mechanism, some components could prove not adequate with respect to the loads. Hence, the maximum instantaneous reaction force and moment of the $j$-th joint, $F_{j}$ and $M_{j}$ respectively, are monitored:

$$
F_{j}=\max _{\hat{t}, w}\left\{\left(\left|\mathbf{F}_{j}(\hat{t})\right|\right)_{w}\right\} ; M_{j}=\max _{\hat{t}, w}\left\{\left(\left|\mathbf{M}_{j}(\hat{t})\right|\right)_{w}\right\} ; j=1, . ., J ; w=1, . ., W
$$

where $\left|\mathbf{F}_{j}(\hat{t})\right|$ and $\left|\mathbf{M}_{j}(\hat{t})\right|$ are the magnitudes of the $j$-th joint reaction force and moment, respectively, computed at the $\hat{t}$-th time step of simulation and related to the $w$-th operating task. Section 2.3 will describe how these scalar quantities are combined in a single parameter in order to simplify the comparison between the different mechanism balanced variants.

Detailed explanations of the algorithm steps are provided in the following subsections.

\subsection{Phase 1: analysis of the unbalanced mechanism operation (Proc-1)}

The working performance of the unbalanced $(U B)$ mechanism, in terms of motor loads and joint reactions, is taken as reference to assess the suitability of possible balancing solutions.

The inverse dynamics problem of the $U B$ mechanism is numerically solved for a proper set of prescribed trajectories, by using the Lagrange equations of motion (see Appendix A.1 for a complete description of the adopted formalism). The dynamic loads acting on the system for operation with the prescribed trajectories are determined by solving (Fig. 1, Proc-1): 


$$
Q_{l}=\frac{d}{d t}\left(\frac{\partial T_{U B}}{\partial \dot{q}_{l}}\right)-\frac{\partial T_{U B}}{\partial q_{l}}+\frac{\partial V_{U B}}{\partial q_{l}}+\frac{\partial \Phi^{T}}{\partial q_{l}} \lambda \quad ; \quad I=1, . ., 6 L
$$

The set of trajectories consists of the most demanding tasks possibly performed by the robot, i.e. those generating the highest dynamic loads. Such trajectories must be provided as inputs to the procedure, since they depend on the specific application. The following results are determined and saved for the subsequent steps of the procedure.

- First performance indicator, QTR ${ }_{U B}$, computed according to Eq. (1).

- Second performance indicator, QIT UB, computed according to Eq. (2).

- Parameters related to the third criterion, $F_{j, U B}$ and $M_{j, U B}$ respectively, computed according to Eq. (3).

- Tasks generating the highest RMS value of the torque on the $n$-th actuator, referred to as critical RMS task and denoted by superscript "CRT", to be adopted in the optimization process for computing the objective function.

- Tasks generating the highest peak (PK) value of the torque magnitude on the $n$-th actuator, referred to as critical PK task and denoted by superscript "CPT" to be adopted in the optimization process for determining the constraints.

It is worth noting that up to $N$ CRT and $N$ CPT different tasks may be found, since they are not necessarily the same for all the actuators.

\subsection{Phase 2: definition and assessment of the balanced variants (Proc- $2 \div$ Proc-8)}

This work studies balancing devices based on counterweights and/or springs and arranged without the installation of auxiliary linkages, in order to increase their practical feasibility.

Figure 2 shows the parameterization adopted for generic balancing elements, namely a counterweight rigidly connected to the $i$-th member and a spring connecting the $i$-th and the $s$-th members.

The $i$-th counterweight appended to the $i$-th member is described by its mass parameter, $m_{i}$, and the position of its CoM, given by vector $\mathbf{r}_{i}$, expressed in the local coordinate system $\sigma_{i}$. Assumptions on the counterweight mass distribution are required for determining the corresponding inertia tensor $\mathbf{J}_{i}$, expressed in $\sigma_{i}$. In this study, cylindrical shaped counterweights are considered, characterized by a fixed ratio $(\tau)$ of radius to thickness and oriented with the axis parallel to the $z_{i}$-axis. Hence $J_{i}$ depends only on the balancing parameters $m_{i}$ and $\mathbf{r}_{i}$, and on two constant scalars, namely $\tau$ and the material density.

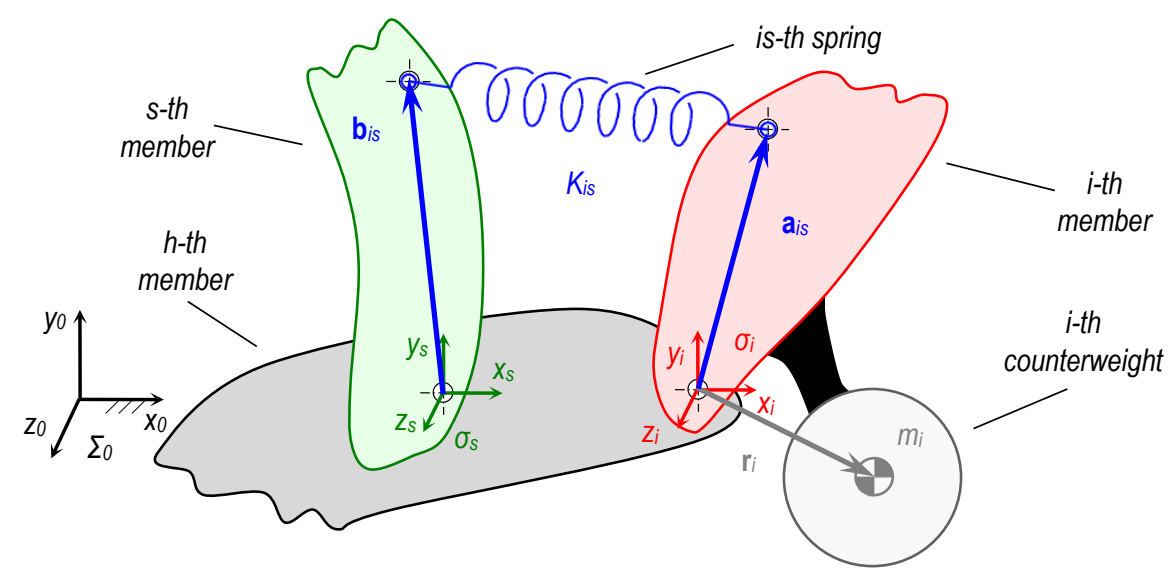

Figure 2. Parameterization of the balancing elements.

The kinetic energy, $T_{\text {mass }}$, and the gravity potential energy, $V_{\text {mass, }}$ associated with the introduction of counterweights are, respectively: 


$$
\begin{gathered}
T_{\text {mass }}=\sum_{i=1}^{L} \frac{1}{2} m_{i} \dot{\mathbf{r}}_{i, 0}^{T} \dot{\mathbf{r}}_{i, 0}+\frac{1}{2} \boldsymbol{\omega}_{i}^{T} \mathbf{J}_{i} \boldsymbol{\omega}_{i} \\
V_{\text {mass }}=\sum_{i=1}^{L}-m_{i} \mathbf{g}_{0}^{T} \mathbf{r}_{i, 0}
\end{gathered}
$$

where $\dot{\mathbf{r}}_{i, 0}$ is the velocity vector of the $i$-th counterweight CoM, $\boldsymbol{\omega}_{i}$ is the angular velocity of the $i$-th member expressed in its body-fixed coordinate system, $\mathbf{g}$ is gravity acceleration vector, the subscript " 0 " means that vectors are expressed in the base reference frame $\Sigma_{0}$ (in particular, $\mathbf{r}_{i, o}={ }^{0} \mathbf{T}_{i} \mathbf{r}_{i}$ ), and $m_{i}=0$ if there is no counterweight appended to the $i$-th member.

The balancing spring connecting members $i$ and $s$ is modelled as a massless zero-free-length extension spring characterized by its stiffness parameter, $K_{i s}$, and by the position of its anchor points, given by vectors $\mathbf{a}_{i s}$ and $\mathbf{b}_{i s}$, expressed in the local coordinate systems of the $i$-th and $s$-th members respectively (Fig. 2 ). The assumption of negligible spring mass and inertia is generally acceptable for common applications [6, 14, 30]. Moreover, it is possible to make normal springs work with zero-free-length behavior (e.g. [13, 31, 32]). The elastic potential energy given by the installation of springs, $V_{\text {spring, }}$ is:

$$
V_{\text {spring }}=\sum_{\substack{i, s=1 \\ i>s}}^{L} \frac{1}{2} K_{i s}\left(\mathbf{a}_{i s, 0}-\mathbf{b}_{i s, 0}\right)^{T}\left(\mathbf{a}_{i s, 0}-\mathbf{b}_{i s, 0}\right)
$$

where the position vectors of the spring anchor points, $\mathbf{a}_{i s, 0}$ and $\mathbf{b}_{i s, 0}$, are expressed in the base reference frame $\Sigma_{0}$ by means of the corresponding coordinate transformation matrices (namely, $\mathbf{a}_{i s, o}={ }^{0} \mathbf{T}_{i} \mathbf{a}_{i s}$ and $\mathbf{b}_{i s, o}={ }^{0} \mathbf{T}_{s} \mathbf{b}_{i s}$ ) and $K_{i s}=0$ if there is no spring connecting to the $i$-th and $s$-th members.

The contributions of the balancing elements modify Eq. (4) as follows

$$
Q_{1}=\frac{d}{d t}\left(\frac{\partial\left(T_{U B}+T_{\text {mass }}\right)}{\partial \dot{q}_{l}}\right)-\frac{\partial\left(T_{U B}+T_{\text {mass }}\right)}{\partial q_{l}}+\frac{\partial\left(V_{U B}+V_{\text {mass }}+V_{\text {spring }}\right)}{\partial q_{l}}+\frac{\partial \Phi^{T}}{\partial q_{l}} \lambda ; I=1, . ., 6 L
$$

Static balancing is achieved if the total potential energy, given by the sum of $V_{U B}, V_{\text {mass }}$ and $V_{\text {spring, }}$, keeps constant for any assumable configuration, i.e. all its partial derivatives with respect to a proper set of independent generalized coordinates $\hat{\mathbf{q}}$ are identically null [8]. In practice, the balancing parameters (i.e. all the parameters describing the balancing counterweights and springs) can be determined by imposing such condition on $N$ of the partial derivatives of the total potential energy appearing in Eq. (8), computed with respect to $\hat{q}$ (see Appendix A.2).

It is worth noting that all the balancing solutions including only springs will be equivalent from the point of view of required motor loads, due to the assumption of negligible spring mass. Conversely, joint reactions depend on the specific spring arrangement.

The proposed procedure generates the set of all the possible mechanism variants that can be arranged with permutations of counterweights and springs (Fig. 1, Proc-2, the e-th generic variant being referred to as $V A R e)$, that satisfy the condition

$$
d=\operatorname{card}\left\{m_{i} \neq 0 ; i=1, . ., L\right\}+\operatorname{card}\left\{K_{i s} \neq 0 ; i, s=1, . ., L ; i<s\right\} \leq D_{\max }
$$

where $d$ is the actual number of balancing elements installed on the mechanism, and $D_{\max }\left(\in \mathbb{N}_{\neq 0}\right)$ is set arbitrarily as an input of the balancing problem. $D_{\max }$ represents the maximum number of balancing elements to be possibly installed on the mechanism; the designer's choice may depend on many qualitative/quantitative criteria (e.g. total cost of the system modifications/additional components, or possible problems of interference between the balancing elements and the mechanism members). The 
minimum value of $D_{\max }$ is equal to 1 , whereas its maximum (within the proposed algorithm) is given by the sum of the mechanism moving members and all the possible pairs of members (also including the fixed frame, see Appendix A.3) ${ }^{1}$. Certainly, having some guidelines for choosing a "reasonable" value for $D_{\max }$ would be convenient. For some architectures it might be possible to find a relation between the mechanism DOFs and the number of required balancing elements (e.g. for planar serial chains of binary links connected by revolute joints [27]). Nonetheless, for a generic mechanism, the methods and tools currently available in the literature do not allow - a priori - either to assess the existence of feasible balancing solutions with the desired specifications, or to determine the minimum number of elements required to achieve gravity compensation. For instance, in case the installation of auxiliary linkages is a viable option, one single balancing element should be theoretically sufficient, since the vertical coordinate of the mechanism global CoM is the only coordinate affecting the gravitational potential energy, as shown by Herder in [33]. Conversely, if the installation of auxiliary linkages is not allowed (that is the case of the proposed algorithm), a number of required balancing elements greater than one may expected; however, depending on the actual mechanism architecture, such assumption may be not confirmed, as occurring for the palletizer robot analyzed in Sec. 3.2. In the Authors' opinion, having a low number of balancing elements would be convenient, in order to limit the mechanism complexity, hence increasing the practical feasibility. Moreover, the amount of mechanism variants VARe to be evaluated grows very fast with an increment in $D_{\max }$, which heavily extends the computational time (see Appendix A.3). Hence, in light of the above considerations, it is suggested that: (i) the balancing process is started by performing a first run of the algorithm with $D_{\max }=1$; (ii) iteratively, $D_{\max }$ is increased by 1 and the algorithm is repeated, until a satisfactory set of balanced variants is possibly found.

Balancing conditions are imposed on each VARe variant of the set so determined. The following $N$ conditions must be verified for all admissible values of $\hat{\mathbf{q}}$ :

$$
\frac{\partial\left(V_{\text {UB }}+V_{\text {mass }}+V_{\text {spring }}\right)}{\partial \hat{q}_{n}}=0 \quad ; \quad n=1, . ., N
$$

For each VARe variant, the procedure searches for an analytical solution to Eq. (10) by using the Matlab ${ }^{\circledR}$ symbolic solver (Fig. 1, Proc-3), which results in a set of analytical relations between the unknowns (see Appendix A.2). If a solution is found ${ }^{2}$, the VARe variant is identified as a possible balanced variant and enters an iterative numerical optimization process, otherwise it is discarded (Fig. 1, Proc-4). The solution of Eq. (10), namely the analytical expressions of the balancing parameters that make the total potential energy invariant, is included in the constraints of the optimization problem. Indeed, at this step no numerical values are assigned to the balancing parameters yet.

As previously illustrated, the primary objective of the study is reducing the system energy consumption. Therefore, a "global" optimality criterion, related to the mechanism energy requirements for the set of trajectories given in input, is adopted. Consistently with the considerations discussed at be beginning of Section 2, optimization of the $c$-th balancing solution (referred to as $S B C$ ) is performed by minimizing an objective function based on the RMS value of the motor actions (Fig. 1, Proc-5). At the first iteration, such function is defined as

\footnotetext{
${ }^{1}$ It is worth noting that the upper limit for $D_{\max }$ is not inherent in the balancing problem, but is a characteristic of the proposed algorithm, which does not admit multiple counterweights attached to a single member or multiple springs connecting a pair of members. In general, the upper (theoretical) limit would be $+\infty$. Furthermore, if a balanced variant with $d$ elements can be found, reasonably a solution with a higher number of balancing elements should also exist [27].

${ }^{2}$ It is worth noting that balancing solutions with the desired design (combination of springs and counterweights without auxiliary linkages) may not exist at all. In addition, the symbolic computation of Eq. (10) can be demanding and, in case the solver fails, some possible balancing solutions may be neglected (see Appendix A.2).
} 


$$
\operatorname{RMS}^{*}\left(\mathbf{x}_{S B C}\right)=\sqrt{\frac{1}{N} \sum_{n=1}^{N}\left(R M S_{S B C, n}^{C R T}\right)^{2}}
$$

where $\mathbf{x}_{S B C}=\left\{m_{i}, \mathbf{r}_{i}, K_{i s}, \mathbf{a}_{i s}, \mathbf{b}_{i s}\right\}^{T}$ is the vector of the unknown balancing parameters $(i, s=1, . ., L, i>S)$, and $R M S_{S B C, n}^{C R T}$ is the RMS value of the $n$-th motor torque, $Q_{n}$, computed for the $C R T$ task defined in Sec. 3.1 by using simplified equations of motion:

$$
Q_{n}=\frac{d}{d t}\left(\frac{\partial\left(T_{U B}+T_{\text {mass }}\right)}{\partial \dot{q}_{n}}\right)-\frac{\partial\left(T_{U B}+T_{\text {mass }}\right)}{\partial q_{n}}+\frac{\partial\left(V_{U B}+V_{\text {mass }}+V_{\text {spring }}\right)}{\partial q_{n}} ; n=1, . ., \mathrm{N}
$$

The following constraint equations must be satisfied, in addition to Eq. (10):

$$
\left\{\begin{array}{l}
\mathbf{x}_{S B C, \min } \leq \mathbf{x}_{S B C} \leq \mathbf{x}_{S B c, \max } \\
P K_{S B C, n}^{C P T} \leq P K_{\max , n}
\end{array}\right.
$$

where $\mathbf{x}_{S B C, \min }$ and $\mathbf{x}_{S B C, \max }$ are the lower and upper bounds for the unknowns, respectively, $P K_{\max , n}$ is the maximum allowed load for the $n$-th actuator and $P K_{S B C, n}^{C P T}$ is the PK value of the $n$-th motor torque, $Q_{n}$, computed for the CPT task defined in Sec. 2.1 by using Eq. (12). Previous investigations had proven the quadratic mean of RMS values more effective than other tested criteria (e.g. the total RMS value or the simple mean of the critical RMS) for effectively reducing the required motor actions [30]. A constrained nonlinear multivariable problem is therefore defined [34]. The unknowns are computed by means of the interior-point method [35], namely by using the "fmincon" Matlab ${ }^{\circledR}$ function.

The numerical values of the balancing parameters for each feasible balancing solution, $\mathbf{x}_{S B, \text { opt, }}$ are therefore determined. It is worth noting that some possible balancing solutions may be discarded also at this step of the procedure (Fig. 1, Proc-6), in case they can not satisfy the constraints given by Eq. (13) and Eq. (14), or convergence is not achieved by the numerical solver.

The operation of each gravity compensated variant of the mechanism is now assessed by inserting the corresponding computed variables $\mathbf{x}_{S B, \text { opt }}$ into Eq. (8), and simulating the numerical model. Inverse dynamic analysis (equivalent to the one performed on the $U B$ mechanism) is carried out over the whole set of prescribed motions taken into account in Sec. 2.1 (Fig. 1, Proc-7), to determine:

- First performance indicator, $Q T R_{S B C}$, as defined by Eq. (1). It is worth noting that the most demanding task may differ from the one identified for the UB mechanism.

- Second performance indicator, $Q I T_{S B C}$, as defined by Eq. (2).

- Joint reaction forces and moments, $F_{j, S B c}$ and $M_{j, S B c}$, as defined by Eq. (3).

- Possible new critical RMS tasks (i.e. different from the ones characterizing the UB mechanism).

- Possible new critical PK tasks.

If at least one new critical RMS or PK task is found, the objective function and/or the constraints equations are updated accordingly (Fig. 1, Proc-8) and a new optimization step is carried out, followed by a new assessment of the dynamic operation of the $S B C$ variant. The iterative loop permits to optimize the balancing solution not only for a single specific task, but for the general operation of the system.

\subsection{Phase 3: ranking of the balancing solutions (Proc-9)}

In order to compare the performance of the balanced variants with the operation of the original mechanism, according to the three criteria previously defined, three non-dimensional parameters are computed. The percentage reduction in the performance indicator $Q R T$ of the $S B C$ variant with respect to the $U B$ mechanism, $\triangle Q R T_{S B C}$, is computed as

$$
\Delta Q R T_{S B C}=\frac{Q R T_{S B C}-Q R T_{U B}}{Q R T_{U B}} \cdot 100
$$


An improved behavior is achieved with the $S B C$ variant if $\triangle Q R T_{S B C}<0$, and the lower is the percentage (up to a minimum of $-100 \%)$, the better is the performance.

The percentage reduction in the performance indicator QIT of the $S B C$ variant with respect to the UB mechanism, $\triangle Q I T_{S B C}$, is defined as

$$
\Delta Q I T_{S B C}=\frac{Q I T_{S B C}-Q I T_{U B}}{Q I T_{U B}} \cdot 100
$$

Like for the previous parameter, the $S B C$ variant provides an enhanced performance if $\Delta Q I T_{S B C}<0$, and the lower is better (maximum reduction for a value of $-100 \%$ ).

Regarding the third criterion, for the $j$-th joint of the $S B C$ variant, the percentage variations of its reaction force and moment, $\Delta F_{j, S B c}$ and $\Delta M_{j, S B c}$ respectively, with respect to the $U B$ mechanism are computed:

$$
\begin{aligned}
\Delta F_{j, S B C} & =\frac{F_{j, S B C}-F_{j, U B}}{F_{j, U B}} \cdot 100 \\
\Delta M_{j, S B C} & =\frac{M_{j, S B C}-M_{j, U B}}{M_{j, U B}} \cdot 100
\end{aligned}
$$

where $j=1, . ., J$. The parameters defined by Eq. (17) and Eq. (18) are intended to detect only possible detrimental effects brought about by the balancing elements, in terms of increments in the joint reactions. Hence, negative values (indicating reductions in the joint reactions) are neglected by setting them to zero (i.e. $\Delta F_{j, S B c}$ and $\Delta M_{j, S B c}$ are forced to assume only positive or null values), since negative variations may conceal the worsening effects exhibited by the other joints. The use of percentage variations permits to combine forces and moments in a single parameter:

$$
\Delta R_{S B C}=\sum_{j=1}^{J} \gamma_{F j} \cdot \Delta F_{j, S B C}+\gamma_{M j} \cdot \Delta M_{j, S B C}
$$

where the weighting factors $\gamma_{F j}$ and $\gamma_{M j}$, for forces and moments respectively, defined as

$$
\begin{gathered}
\gamma_{F j}=\frac{F_{j, U B}}{\sum_{j=1}^{J} F_{j, U B}} \\
\gamma_{M j}=\frac{M_{j, U B}}{\sum_{j=1}^{J} M_{j, U B}}
\end{gathered}
$$

are adopted to emphasize the variations of the highest loads (since a very low reaction would remain negligible even in case of a high percentage variation). The lower is the parameter $\Delta R_{S B C}$ (minimum value being $0 \%$ ), the better is the performance of the $S B C$ variant. This parameter allows discriminating between the balancing variants featuring only springs. Indeed, such variants necessarily exhibit by the same values of both $\triangle Q R T_{S B C}$ and $\triangle Q I T_{S B C}$ (since they are dynamically equivalent under the assumption of massless springs), whereas significant differences in the joint reactions may exist, depending on the spring arrangements.

The balanced variants are ranked by using the three non-dimensional parameters, according to the different priority of the performance indicators, i.e. in order of best $\triangle Q R T_{S B C}$, then best $\triangle Q I T_{S B C}$, and finally best $\Delta R_{S B C}$ (Fig. 1, Proc-9). The procedure provides both a global ranking of all the balancing solutions and a 
separate ranking for each class of balanced variants (i.e. only-counterweights, only-springs and hybrid solutions). Indeed, although the proposed quantitative criteria can provide indications on the dynamic operation of each mechanism variant independently of the specific type/arrangement of its balancing elements, a direct comparison between different classes of balanced variants may be considered not completely rigorous.

It is worth noting that the adopted performance indicators do not provide a full insight into the actual load condition of each actuator and/or joint. However, they were preferred to more complex parameters for obtaining a simple and concise overview of the dynamic performance of all the balanced variants.

Finally, it is important to remark that the proposed criteria are only intended for providing a quantitative assessment of the expected working performance of the identified balanced variants, for a preliminary selection of the most promising ones. Then, additional criteria - other quantitative indicators (e.g. implementation and maintenance costs and/or risk indicators for safety assessment) and/or qualitative parameters (e.g. ergonomic features, marketing strategies) and/or the common practice/internal guidelines of designers/manufacturers - might be evaluated in order to determine the most convenient solution.

\section{Case study: industrial palletizing robot}

The balancing procedure proposed in Sec. 2 was adopted for the gravity compensation of an industrial robotic arm, namely the 4-DOF palletizing robot here analyzed. The manipulator was characterized by rather heavy moving members and operation with low/moderate velocities and accelerations. Given these characteristics, gravity compensation appeared a viable strategy to significantly reduce the motor loads of the manipulator, hence solving its overload and power consumption issues.

Figure 3 shows the robot kinematic scheme. Actuator 1 rotates the moving links around a fixed vertical axis. A planar 2-DOF pantograph-like linkage, driven by two actuated prismatic joints (Actuator 2 and Actuator 3, each one composed of a linear guide and a ball screw driven by a brushless servo motor with timing belt transmission), moves the end effector carrier on a vertical plane. Actuator 2 provides translation along the horizontal direction, with a transmission ratio of +6 between the slider of the horizontal linear guide (member 2, Fig. 4) and the end effector carrier. Similarly, Actuator 3 controls the vertical translation, with a transmission ratio of -5 . Actuator 4 , mounted on the end effector carrier, controls the gripper rotation. A further double parallelogram linkage imposes vertical orientation on the gripper rotational axis, which is a solution frequently adopted in palletizing robots.

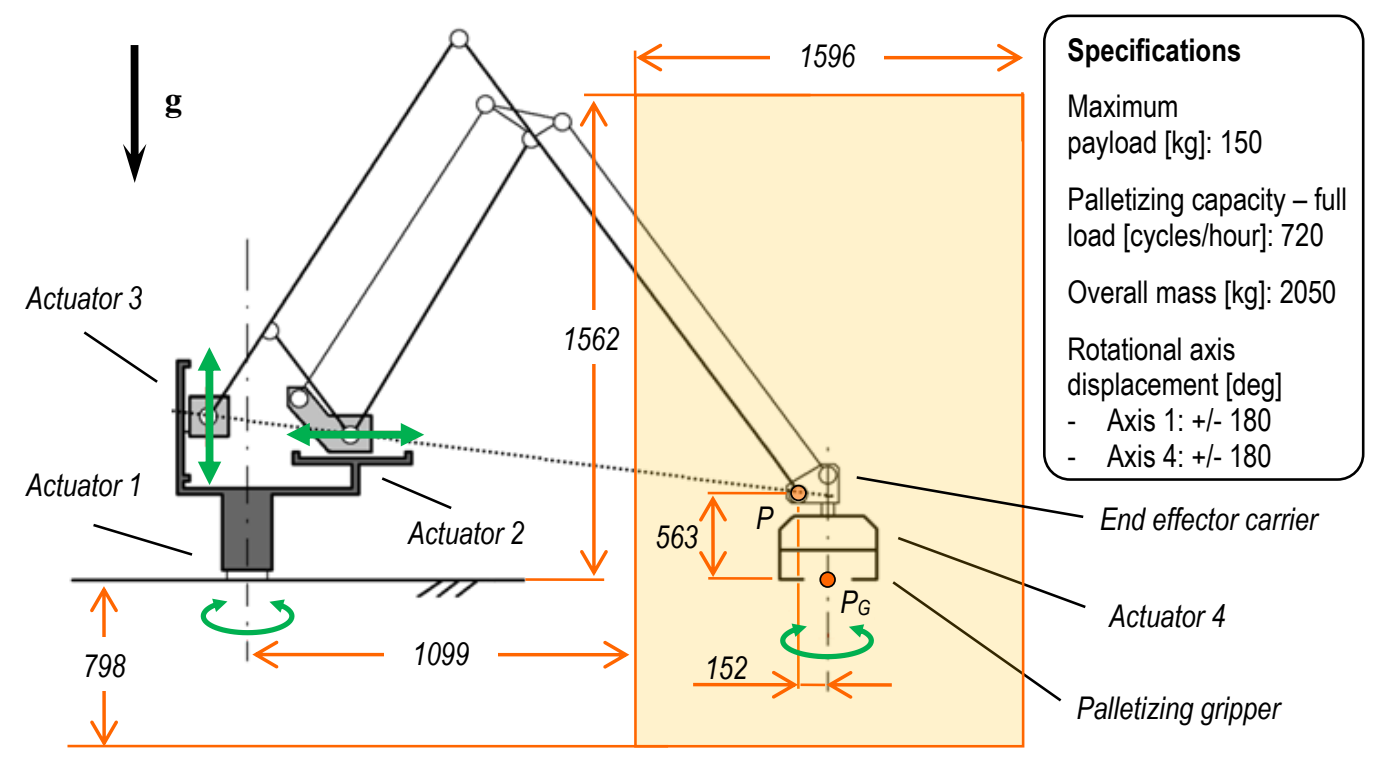

Figure 3. Schematics of the palletizing manipulator: workspace referred to the gripper reference point 


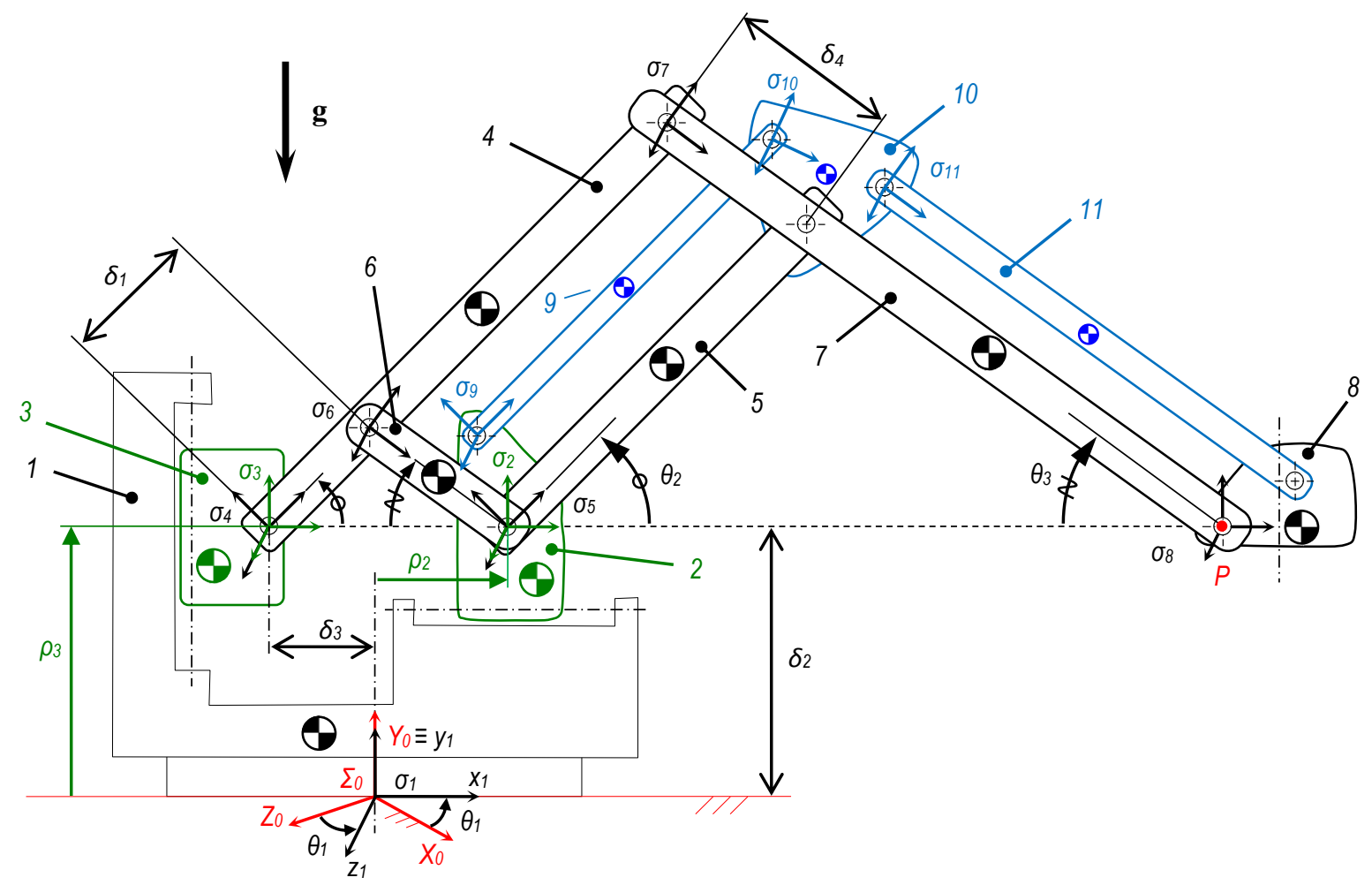

Figure 4. Definition of coordinate systems and kinematic parameters.

Figure 4 shows the coordinate systems and the main kinematic parameters defined for the analysis. The coordinate system $\Sigma_{0} \equiv X_{0} Y_{0} Z_{0}$ is established on the fixed base, with the $Y_{0}$-axis parallel to the gravity vector, g. The Cartesian frame $\sigma_{1} \equiv x_{1} y_{1} z_{1}$, attached to member 1 , is established with the same origin of $\Sigma_{0}$ and the $y_{1}$-axis coincident with the $Y_{0}$-axis. The rotation of the moving members around the vertical axis is described by the angle $\theta_{1}$. The $x_{1} y_{1}$-plane of each Cartesian frame $\sigma_{l}$, attached to the $l$-th member, is parallel to the $x_{1} y_{1}$-plane. The coordinate systems $\sigma_{2} \equiv x_{2} y_{2} z_{2}$ and $\sigma_{3} \equiv x_{3} y_{3} z_{3}$, attached to the two sliders, members 2 and 3 respectively, are oriented like $\sigma_{1}$. The horizontal translation of $\sigma_{2}$ and the vertical translation of $\sigma_{3}$, with respect to $\sigma_{1}$, are described by the variables $\rho_{2}$ and $\rho_{3}$ respectively, whereas the constants $\delta_{2}$ and $\delta_{3}$ give their corresponding vertical and horizontal offsets.

The orientation of all moving members on plane $x_{1} y_{1}$, is described by the two angles $\theta_{2}$ and $\theta_{3}$, defined with respect to the $x_{1}$-axis. The rotation of the palletizing gripper is not taken into account, as explained in Sec. 3.1.

The solution of direct kinematics, providing the position vector $\left(p_{x}, p_{y}, p_{z}\right)$ of the reference point $P$ of the end effector carrier as function of the independent generalized coordinates $\theta_{1}, \rho_{2}$ and $\rho_{3}$, is given by:

$$
\left\{\begin{array}{l}
p_{x}=\left(6 \rho_{2}+5 \delta_{3}\right) \cos \theta_{1} \\
p_{y}=-5 \rho_{3}+6 \delta_{2} \\
p_{z}=-\left(6 \rho_{2}+5 \delta_{3}\right) \sin \theta_{1}
\end{array}\right.
$$

The angles $\theta_{2}$ and $\theta_{3}$ can be inferred from the following conditions:

$$
\left\{\begin{array}{l}
\delta_{1} \cos \theta_{2}+\delta_{4} \cos \theta_{3}=\rho_{2}+\delta_{3} \\
\delta_{1} \sin \theta_{2}-\delta_{4} \sin \theta_{3}=-\rho_{3}+\delta_{2}
\end{array}\right.
$$

Since the manipulator featured actuated prismatic joints and hybrid serial-parallel kinematics, strategies commonly adopted to balance serial manipulators were not directly applicable. Moreover, multiple options 
for achieving gravity compensation were found in the first phase of the study. Because of these factors, this application was considered particularly suitable to prove the effectiveness of the implemented procedure.

The algorithm was executed (setting $D_{\max }=2$ ) on a consumer laptop (Intel i7-3612QM CPU @ $2.1 \mathrm{GHz}$, RAM $8 \mathrm{~GB}$ ) requiring a total computational time of about 30 minutes, with about 10 minutes being required for completing Proc-2 and Proc-3.

\subsection{Balancing problem and simulated tasks}

Actuators 1 and 4 provide rotation around vertical axes, and therefore they are not affected by gravity loads. However the torque of Actuator 1 may be significantly affected by the introduction of balancing devices attached to the robot moving parts. Hence Actuator 1 is taken into account in the dynamic model for the balancing process. Conversely Actuator 4 is neglected, since it is only affected by the payload carried by the gripper. The orientation of the gripper is considered fixed and its mass and inertia are added to those of the end effector carrier.

The proposed method is used to generate balancing solutions including a maximum of two elements, attached to members $1 \div 7$. Since the balancing problem is planar, the vectors locating the counterweight CoMs and the spring anchor points are expressed as functions of two parameters:

$$
\begin{aligned}
& \mathbf{r}_{i}=r_{i} e^{i \varphi_{i}} \\
& \mathbf{a}_{i s}=a_{i s} e^{i \alpha_{i s}} \\
& \mathbf{b}_{i s}=b_{i s} e^{i \beta_{i s}}
\end{aligned}
$$

where $i, s=1, . ., 7$ (with $i>s$ ), $r_{w i}, a_{s i k}, b_{s i k}$ are the magnitudes and $\varphi_{i}, \alpha_{i s}, b_{i s}$ are the angles describing the orientation with respect to the $x$-axis of the corresponding coordinate systems (namely $\sigma_{i}$ for $\mathbf{r}_{i}$ and $\mathbf{a}_{i s}, \sigma_{s}$ for $\mathbf{b}_{\text {is }}$ ).

In the case of a spring connecting two adjacent members (i.e. spanning over one single joint), equivalent spring arrangements (in terms of elastic potential energy) can be obtained by simply varying the spring angles $\alpha_{i s}$ and $\beta_{i s}$ by the same quantity (hence keeping constant the difference $\alpha_{i s}-\beta_{i s}$ ), while maintaining unaltered the other parameters $K_{i s}, a_{i s}$ and $b_{i s}$ [27]. Consequently, one angle can be arbitrarily chosen and the condition $\alpha_{i s}=0$ is set.

Since palletization is basically a pick-and-place task, point-to-point trajectories between all the possible couples of points of a discrete spatial grid, into which the workspace is divided, are considered when solving inverse dynamics. In particular, a total of 343 grid points is considered (Fig. 5a), given by: 7 by 7 regular grids covering the rectangular sections of the workspace on vertical planes passing through axis $Y_{0}$ (including boundaries); 6 angular divisions with angles of $\pi / 6$ around axis $Y_{0}$ in the interval $[0, \pi]$. The system symmetry is exploited to take into account only half the workspace, since in real industrial layouts the palletizing tasks between two loading/unloading stations seldom exceed a rotation of $\pi$ around $Y_{0}$.

Motion is imposed on each actuator by using a single quintic polynomial function that prescribes the displacement between two grid points in a fixed time interval of $2.5 \mathrm{~s}$ (namely, half the duration of a working cycle at the maximum palletizing capacity). The generic law of motion in terms of angular position, velocity and acceleration is shown in Fig. 5b. 
(a)

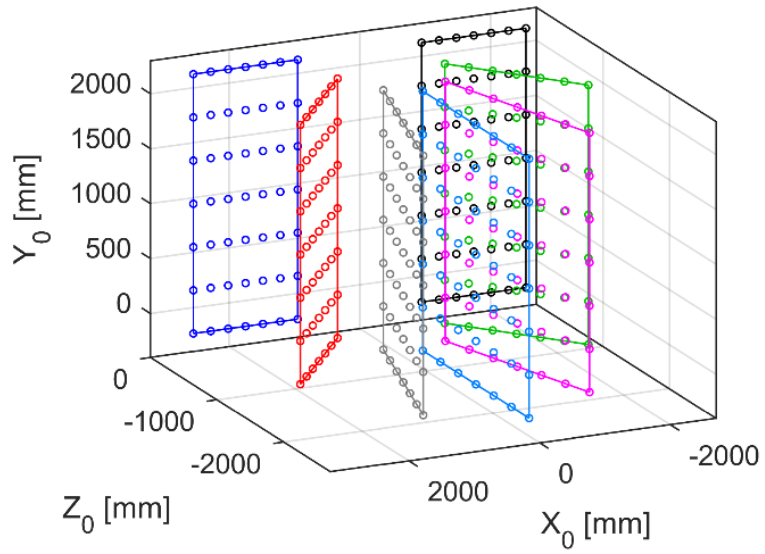

(b)

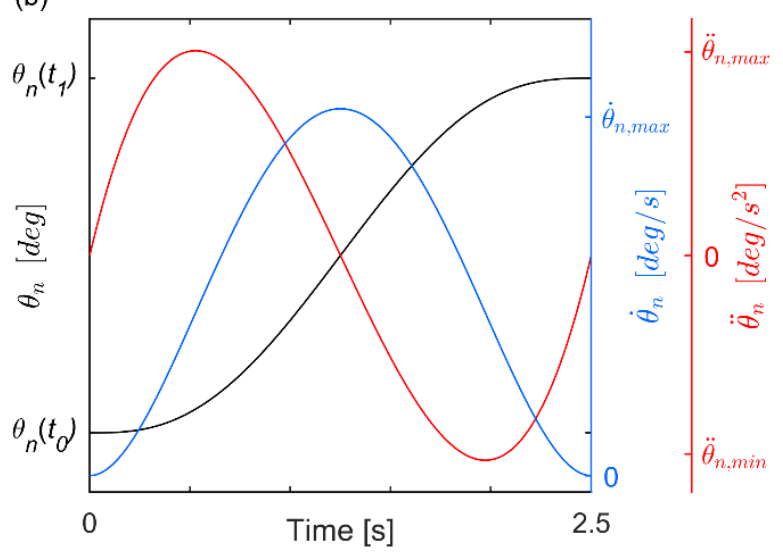

Figure 5. (a) Discrete grid of points for inverse dynamics, (b) generic angular position $\left(\theta_{n}\right)$, velocity $\left(\dot{\theta}_{n}\right)$ and acceleration $\left(\ddot{\theta}_{n}\right)$ law of motion (actual values depend on both the actuator and the trajectory).

\subsection{Feasible balanced variants}

A total of 406 variants is generated and evaluated (see Appendix A.3). Due to the pantograph-like architecture of the robot (which features many similar triangles), dozens of variants providing exact static balancing are found by the algorithm. The set of possible balanced variants also includes known solutions requiring only one counterweight [36,37]. However, only eleven variants satisfy the chosen optimization constraints (e.g. moderate mass increment and acceptable spring rate), whereas all the others are discarded.

Table 1 reports the feasible solutions generated by the gravity compensation procedure and the optimized values of the corresponding balancing parameters. Schematic representations of three variants are shown in Fig. 6, as examples. The high number of possible variants offers a large degree of flexibility for implementing balancing in practice. Many solutions exhibit comparable values and appear potentially profitable. In particular, several variants including counterweights (which, in general, can be arranged more easily than springs $[6,14]$ ) would bring an acceptable increment in the robot total mass (below $+20 \%$ ). Hence, the use of quantitative ranking criteria appears essential for choosing the most advantageous solution.

(a)

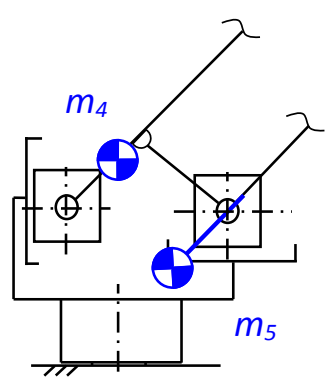

(b)

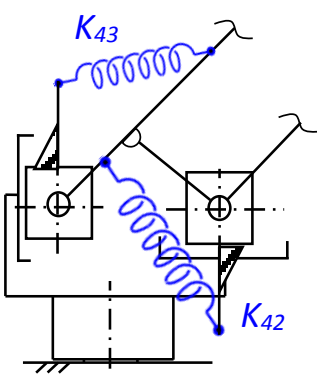

(c)

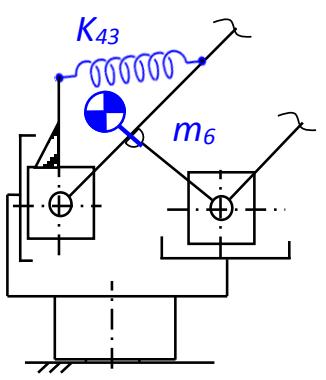

Figure 6. Schematic representation of tree balancing solutions: (a) SB1, (b) SB4, (c) SB10.

Figure 7 shows the balancing variants ranked according to the three criteria proposed in Sec. 2.3, divided by class (only-counterweights, only-springs and hybrid solutions). A remarkable reduction in the estimated energy requirements is achieved with all solutions, thus proving the effectiveness of the proposed approach. The increment in joint reactions is acceptable for all the variants, as the index $\Delta R_{S B}$ keeps below $10 \%$. As expected, the three balanced variants featuring only springs (which are considered massless) exhibit the best performance in terms of motor actions. Four hybrid variants, namely the SB6 and the SB9-11, show a behavior closely comparable to that characterizing the spring-only solutions. Basically, these seven variants can be considered equivalent for the enhancement of motor loads and energy efficiency in dynamic operation. Therefore, they all appear suitable to be arranged on the actual robot. The variant SB10, namely the best hybrid variant, was eventually selected as the most convenient one for 
practical implementation, based on considerations about the limited increment in both the mechanism complexity and its weight (additional mass being less than $10 \%$ of the $U B$ robot mass).

Table 1. Feasible balancing solutions.

\begin{tabular}{|c|c|c|c|c|c|c|c|c|c|}
\hline $\begin{array}{c}\text { balanced } \\
\text { variant }\end{array}$ & param. & $\begin{array}{c}K \\
{[\mathrm{~N} / \mathrm{mm}]}\end{array}$ & $\begin{array}{c}a \\
{[\mathrm{~mm}]}\end{array}$ & $\begin{array}{c}\alpha \\
{[\mathrm{rad}]}\end{array}$ & $\begin{array}{c}b \\
{[\mathrm{~mm}]}\end{array}$ & $\begin{array}{c}B \\
{[\mathrm{rad}]}\end{array}$ & $\begin{array}{c}m \\
{[\mathrm{~kg}]}\end{array}$ & $\begin{array}{c}r \\
{[\mathrm{~mm}]}\end{array}$ & $\begin{array}{c}\varphi \\
\text { [rad] }\end{array}$ \\
\hline \multirow{2}{*}{$S B 1$} & $m_{4}$ & - & - & - & - & - & 228.6 & 81.3 & 0 \\
\hline & $m_{5}$ & - & - & - & - & - & 500.00 & 500.0 & $\pi$ \\
\hline \multirow{2}{*}{$S B 2$} & $m_{4}$ & - & - & - & - & - & 384.7 & 500.0 & $\pi$ \\
\hline & $m_{6}$ & - & - & - & - & - & 234.2 & 500.0 & 0 \\
\hline \multirow{2}{*}{ SB3 } & $K_{43}$ & 5.669 & 998.9 & 0 & 499.9 & $\pi / 2$ & - & - & - \\
\hline & $K_{53}$ & 4.486 & 250.0 & $\pi$ & 499.9 & $\pi / 2$ & - & - & - \\
\hline \multirow{2}{*}{ SB4 } & $K_{42}$ & 5.000 & 250.0 & 0 & 448.5 & $3 / 2 \pi$ & - & - & - \\
\hline & $K_{43}$ & 5.711 & 995.6 & 0 & 497.8 & $\pi / 2$ & - & - & - \\
\hline \multirow{2}{*}{ SB5 } & $K_{42}$ & 4.898 & 476.9 & 0 & 271.1 & $\pi / 2$ & - & - & - \\
\hline & $K_{53}$ & 7.143 & 264.1 & 0 & 499.8 & $\pi / 2$ & - & - & - \\
\hline \multirow{2}{*}{ SB6 } & $m_{4}$ & - & - & - & - & - & 228.6 & 347.1 & 0 \\
\hline & $K_{43}$ & 6.334 & 989.0 & 0 & 486.7 & $\pi / 2$ & - & - & - \\
\hline \multirow{2}{*}{$S B 7$} & $m_{4}$ & - & - & - & - & - & 418.1 & 440.1 & $\pi$ \\
\hline & $K_{53}$ & 4.228 & 250.0 & $\pi$ & 439.0 & $3 / 2 \pi$ & - & - & - \\
\hline \multirow{2}{*}{ SB8 } & $m_{4}$ & - & - & - & - & - & 384.7 & 500.0 & $\pi$ \\
\hline & $K_{42}$ & 3.064 & 250.0 & 0 & 500.0 & $\pi / 2$ & - & - & - \\
\hline \multirow{2}{*}{ SB9 } & $m_{4}$ & - & - & - & - & - & 228.6 & 402.3 & 0 \\
\hline & $K_{52}$ & 7.930 & 800.0 & 0 & 500.0 & $\pi / 2$ & - & - & - \\
\hline \multirow{2}{*}{ SB10 } & $m_{6}$ & & - & - & - & - & 181.5 & 77.8 & $\pi$ \\
\hline & $K_{43}$ & 5.727 & 994.0 & 0 & 497.0 & $\pi / 2$ & - & - & - \\
\hline \multirow{2}{*}{ SB11 } & $m_{6}$ & - & - & - & - & - & 500.0 & 163.0 & 0 \\
\hline & $K_{52}$ & 7.077 & 800.0 & 0 & 500.0 & $\pi / 2$ & - & - & - \\
\hline
\end{tabular}

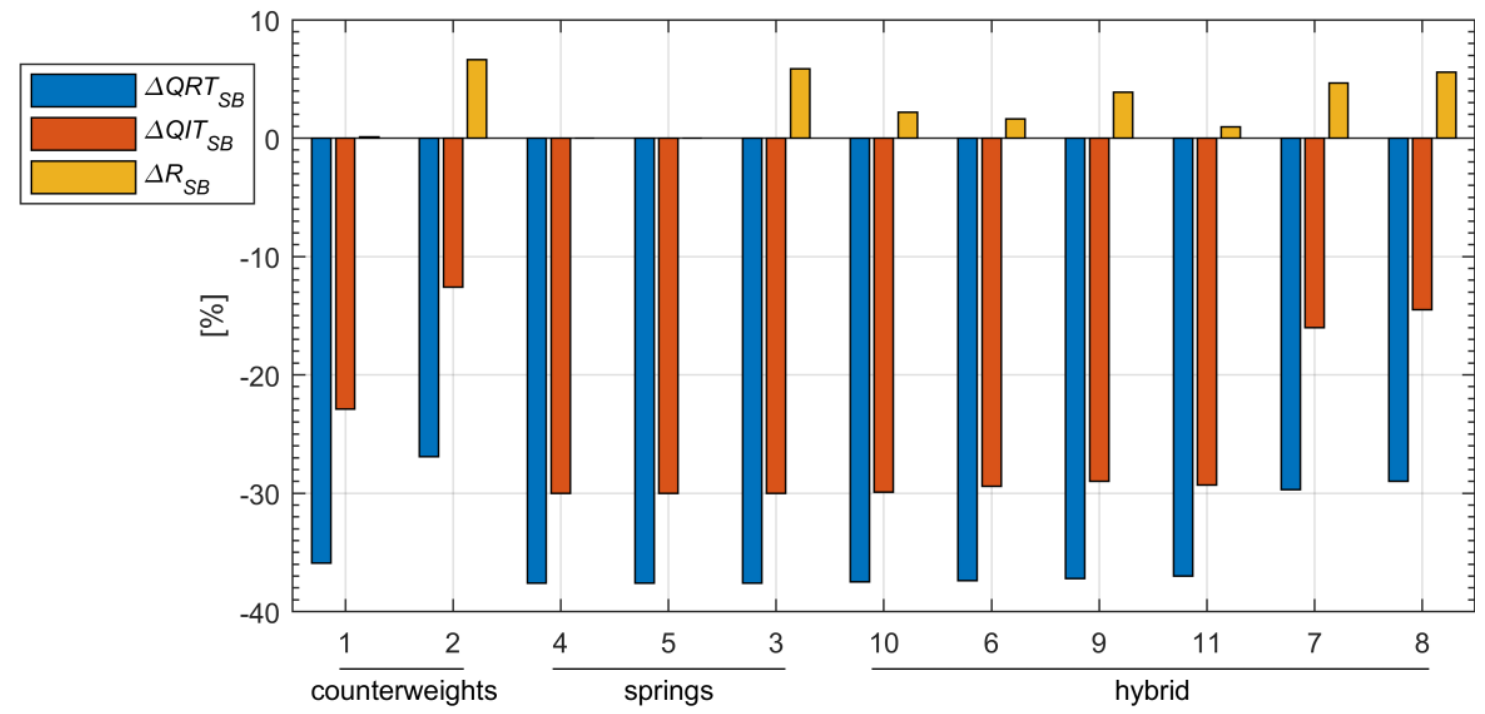

SB variant

Figure 7. Ranking of the balancing solutions, divided by class (best solution on the left side of each group). 


\subsection{Multibody simulations of a real palletizing task}

Numerical models of the $U B$ mechanism and of the best variant for each configuration (namely only springs, $S B 4$, only counterweights, $S B 1$, and hybrid arrangement, $S B 10$, Fig. 6) are implemented by using a commercial multibody software (MSC Adams ${ }^{\mathrm{TM}}$ ) to verify the effectiveness of the proposed procedure ${ }^{3}$.

Simulations are perfomed by imposing the path A-C-B-E-F-D-A (Fig. 8a, referred to as path $\Lambda$ ) on the reference point $P$ of the end effector carrier. Path $\Lambda$ represents a demanding task of the studied robot and has the following main features: rotation of $60^{\circ}$ around the $Y_{0^{-}}$axis; translation of $1046 \mathrm{~mm}$ along the $x_{1^{-}}$ axis, reaching the external boundary of the workspace; translation of $1500 \mathrm{~mm}$ along the $Y_{0}$-axis, to about the upper boundary of the workspace.

(a)

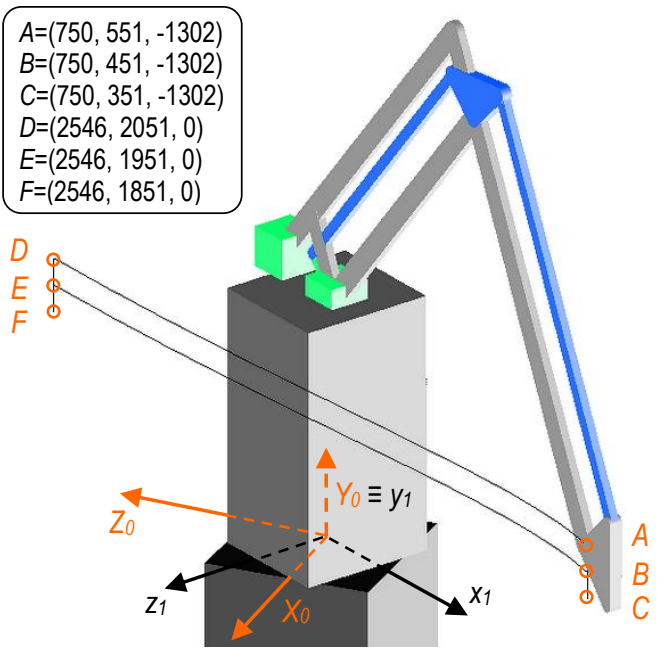

(b)

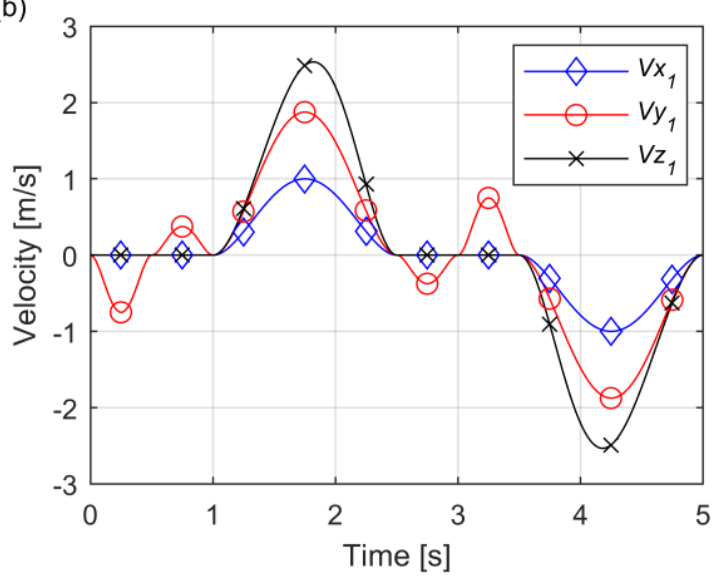

Figure 8. (a) simulated trajectory (point locations in $\Sigma_{0}[\mathrm{~mm}]$ ), (b) velocity of the end effector.

\footnotetext{
${ }^{3}$ Preliminary investigations on the examined manipulator had shown that the mass of suitable balancing springs might be about $40 \mathrm{~kg}$, i.e. only $2 \%$ of the total robot mass [30]. Hence, reasonably, the mass and inertia of the springs are expected to marginally affect the manipulator dynamics, as initially conjectured. In light of these considerations and consistently with the assumptions of the proposed algorithm, the springs are modelled as massless entities also within the multibody environment.
} 

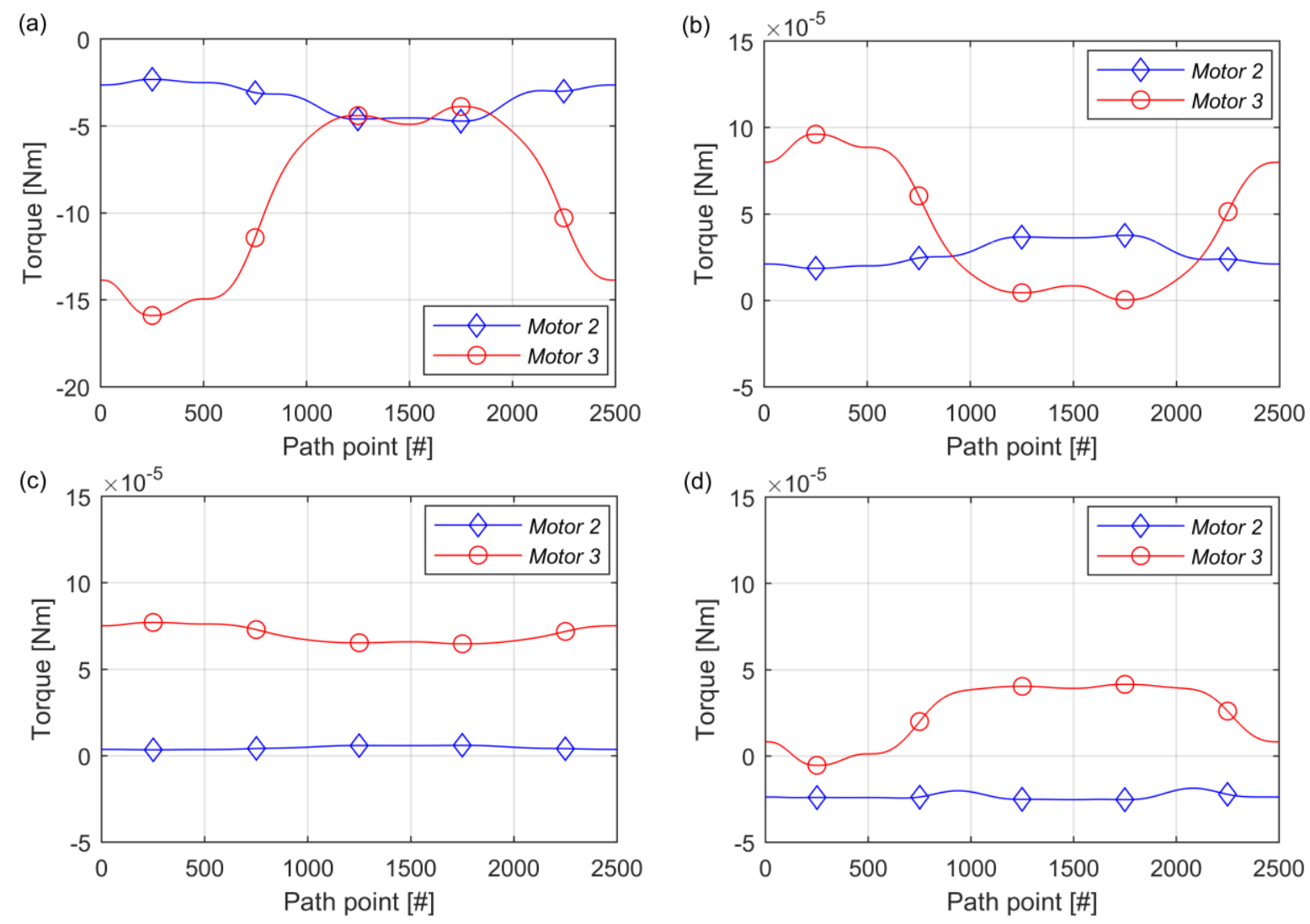

Figure 9. Static torques along path $\Lambda$ : (a) UB, (b) SB1, (c) SB4, (d) SB10.

Two working conditions are simulated for each mechanism. In the first test, point $P$ is driven through path $\Lambda$ in static conditions, i.e. velocities and accelerations are set to zero. The static torques of Motor 2 and Motor 3 (namely, the servo motors driving Actuator 2 and Actuator 3, respectively), required for the robot equilibrium, are computed for 2500 points along the path by taking into account the nominal efficiency and the actual transmission ratio of the corresponding mechanical transmissions.

In the second test, a palletizing cycle is replicated. Path $\Lambda$ is executed in a total time of 5 s, i.e. at the maximum palletizing capacity of the robot. The components of the velocity vector of point $P$, expressed in $\sigma_{1}$, are reported in Fig. $8 \mathrm{~b}$. A payload of $150 \mathrm{~kg}$ (namely, the maximum value of the robot specifications) is loaded at point $C$ and unloaded at point $F$. The required torques for Motor 1-3 are computed with a time step of $0.002 \mathrm{~s}$.

Figure 9 shows the computed static torques. The motor torques required for sustaining the robot own weight are remarkable for the UB mechanism, particularly for Motor 3, which generates the vertical motion of the end effector. Conversely, all the balanced variants exhibit negligible motor loads, torque values being more than five orders of magnitude lower than for the UB mechanism. Reasonably, such small values are ascribable to approximations of the parameters of the models and residuals of the numerical solver. Nonetheless, the results confirm that complete compensation of gravity loads is achieved for all the balanced variants.

The motor torques required for executing the palletizing task are shown in Fig. 10, as functions of time. The RMS values and peak absolute values (|peak|) off all the plotted curves are reported in Table 2 . The percentage variations with respect to the $U B$ mechanism are also tabulated $(\triangle \%)$.

The curves related to Motor 1 are basically superimposed also for SB1 and SB10 variants (Fig. 10a), and the corresponding RMS and | peak | values do not exhibit significant variations (Table 2). This confirms that possible detrimental effects of the optimized counterweights on the first rotational axis are negligible. As for Motor 2 and Motor 3, all the balanced variants are characterized by remarkably lower torques in terms of both RMS and |peak|. Consistently with the ranking reported in Fig. 7, the best overall performance is provided by the SB4 variant, followed by the SB10 and then by the SB1. Therefore, these 
results prove the effectiveness of the proposed procedure, even in case of a variable payload. In particular, the proposed optimization process is confirmed a valid tool to generate convenient balancing solution even in case massive counterweights are required.
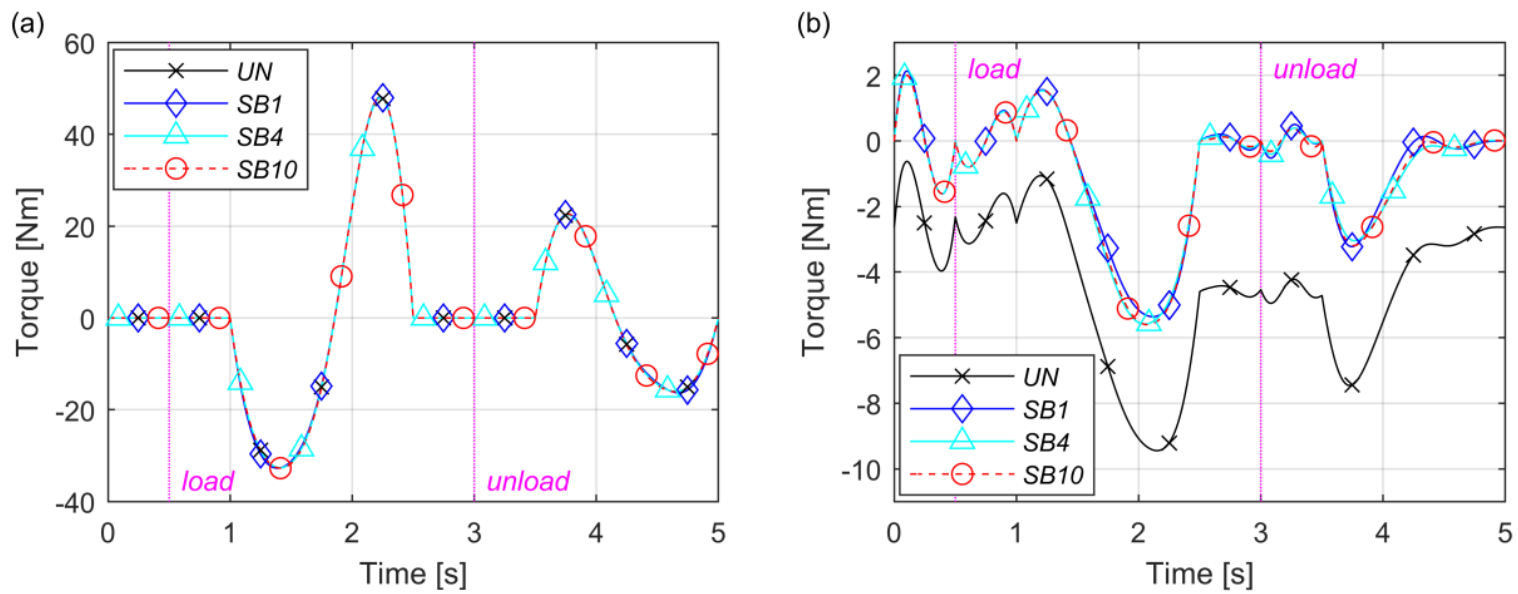

(c)

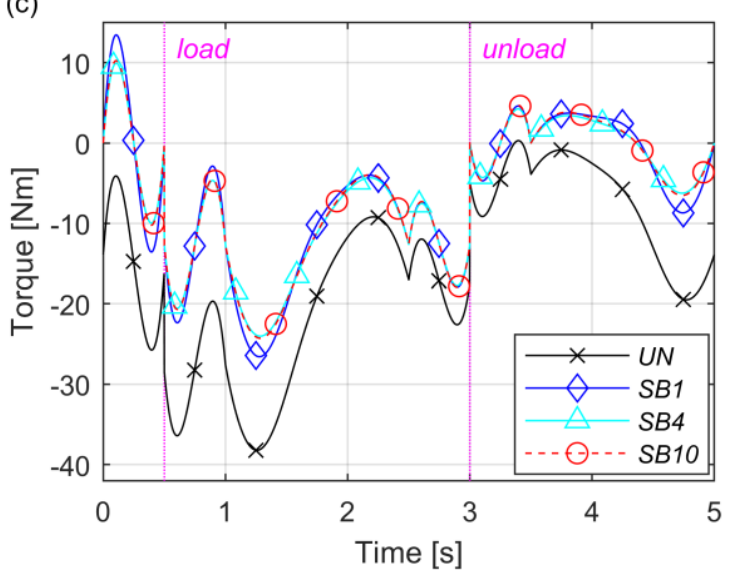

Figure 10. Dynamic torques of the palletizing cycle: (a) Motor 1, (b) Motor 2, (c) Motor 3.

Table 2. Statistics of the torques required for the palletizing task.

\begin{tabular}{ccrrrrrrr}
\hline \multirow{2}{*}{ Motor } & & UB & \multicolumn{2}{c}{$S B 1$} & \multicolumn{2}{c}{$S B 4$} & \multicolumn{3}{c}{$S B 10$} \\
\hline \multirow{2}{*}{1} & & $T[\mathrm{Nm}]$ & $T[\mathrm{Nm}]$ & \multicolumn{1}{c}{$\Delta \%$} & $T[\mathrm{Nm}]$ & \multicolumn{1}{c}{$\Delta \%$} & $T[\mathrm{Nm}]$ & \multicolumn{1}{c}{$\Delta \%$} \\
\hline \multirow{2}{*}{2} & RMS & 17.48 & 17.58 & +0.6 & 17.48 & 0 & 17.56 & +0.4 \\
& Ipeak| & 47.94 & 48.13 & +0.4 & 47.94 & 0 & 48.10 & +0.3 \\
& RMS & 4.98 & 2.05 & -58.8 & 2.12 & -57.4 & 2.13 & -57.2 \\
\multirow{2}{*}{3} & Ipeak| & 9.45 & 5.36 & -43.2 & 5.59 & -40.9 & 5.61 & -40.7 \\
& RMS & 18.98 & 11.26 & -40.7 & 10.55 & -44.4 & 10.63 & -44.0 \\
& |peak| & 38.21 & 26.57 & -30.4 & 24.02 & -37.1 & 24.28 & -36.5 \\
\hline
\end{tabular}

\subsection{Discussion}

The reported results are satisfactory and confirm the effectiveness of the proposed procedure. Firstly, the procedure could generate a wide range of feasible balanced variants, including hybrid counterweight/spring arrangements. Secondly, thanks to optimization, a remarkable improvement in the expected energy efficiency of the robot could be achieved for all the solutions. In particular, a significant enhancement of the robot dynamic performance could be obtained also for a working cycle with variable payload. Thirdly, the adopted quantitative criteria based on the estimated dynamic operation, arbitrarily defined, permitted to compare and rank variants characterized by completely different arrangements of the balancing elements, as well as to assess the actual benefits (in terms of motor loads) and drawbacks (in 
terms of joint reactions) of each variant, with respect to the UB mechanism. In particular, the analysis showed that apparently similar solutions (i.e. characterized by comparable values of their balancing parameters) might exhibit a very different dynamic behavior. For instance, the variants $S B 7$ and $S B 8$, albeit comparable to the other hybrid solutions, would provide worse working performance. Hence, the reduced subset of the most convenient variants could be identified, thus allowing the design process to focus on such variants to implement gravity compensation on the actual robot. This proved the presented procedure as a suitable tool to help the design of statically balanced mechanisms.

\section{Conclusion}

This paper dealt with the gravity compensation of mechanisms characterized by both serial and parallel kinematic chains. A novel approach to automate the generation of feasible static balancing solutions, and the identification of the most convenient ones, was presented. Given a mechanism with known analytically solvable - forward kinematics, and starting from very limited input data concerning the balancing elements to be installed, the procedure searches for possible balanced variants that can be arranged with proper combinations of counterweights and springs, without adding auxiliary linkages (which are sometimes required to achieve balancing solutions). If analytical solutions are found, the balancing parameters are determined by means of a numerical optimization procedure aimed at maximizing the mechanism energy efficiency. The resulting balanced variants are ranked by using three simple quantitative criteria that were specifically developed for guiding the selection of the most suitable solutions. The main merits of the proposed approach can be summarized as follows.

- It is suitable for open-chain, closed-chain and hybrid mechanisms.

- It does not require a priori assumptions on the arrangement/location of the balancing elements.

- It can generate exact balancing solutions with counterweights and springs combined together.

- Each mechanism variant is numerically optimized in terms of expected energy consumption, by taking into account its dynamic operation with typical working conditions.

- It provides an estimate of the dynamic performance of each variant by using three simple indicators.

The proposed approach was successfully adopted for an industrial palletizing robot. On one hand, the procedure could generate a set of possible balanced variants of the manipulator, most of them exhibiting a remarkable reduction in the expected energy consumption. On the other hand, the most convenient variants to be implemented in practice could be identified by using the implemented performance indicators. Hence the proposed method proved as an effective tool for gravity compensation purpose.

\section{Appendix}

\section{A.1. Mechanism kinematics and dynamics}

The proposed method can operate on a generic $N$-DOF mechanism modeled as a chain of $L$ rigid bodies connected by kinematic joints, provided that a closed-form solution for the forward kinematics problem can be found. The benefits of different balancing solutions on the mechanism operation are assessed by solving the inverse dynamics problem to compute the dynamic loads acting on the system for a set of prescribed trajectories of interest.

Since the mechanism may feature both open- and closed-chains, the actual model parameterization depends on the specific geometry of the linkage. In general, a local Cartesian coordinate system is attached to each rigid body. Its position and orientation with respect to a global reference frame are defined by means of three Cartesian coordinates and three Euler angles, respectively. Proper homogeneous transformation matrices from the $i$-th local system $\sigma_{i}$ to the global frame $\Sigma_{0},{ }^{0} \mathbf{T}_{i}$, are then defined [38].

At first, the mechanism forward kinematics is solved for determining the pose, velocities and accelerations of its moving parts. These results permit to determine the expressions of the kinetic and the potential energies of each member and of the corresponding balancing elements. The potential energy is utilized for identifying the balanced variants (see Appendix A2 for further details). Then, the kinetic and the potential energies are employed in the Lagrange equations of motion, which are solved to estimate the 
motor actions. The expressions describing the forward kinematics of each mechanism member (as functions of a proper set of independent generalized coordinates $\hat{\mathbf{q}}=\left\{\hat{q}_{n}\right\}^{T}, n=1, . ., N$ ) are therefore assumed as know input data for the procedure that generates balancing solutions.

The following formulation is adopted for writing the Lagrange equations of motion [39]:

$$
\left\{\begin{array}{l}
Q_{l}=\frac{d}{d t}\left(\frac{\partial T}{\partial \dot{q}_{l}}\right)-\frac{\partial T}{\partial q_{l}}+\frac{\partial V}{\partial q_{l}}+\frac{\partial \Phi^{T}}{\partial q_{l}} \lambda ; \quad I=1, . ., 6 L \\
\Phi(\mathbf{q}, t)=0
\end{array}\right.
$$

where $\mathbf{q}=\left\{q_{l}\right\}^{T}$ with $l=1, . ., 6 L$ is the vector of all generalized coordinates for a generic spatial linkage, $Q_{l}$ is the non-conservative applied generalized force related to the $I$-th generalized coordinate, $T$ and $V$ are the total kinetic energy and total potential energy of the mechanism, respectively, $\lambda$ is the vector of (6L-N) Lagrange multipliers, and Eq. (A2) describes the $(6 L-N)$ kinematic constraints ( $\Phi$ denoting the column vector of the algebraic expressions of the kinematic constraints). Hence, friction is neglected in the inverse dynamics problem.

\section{A.2. Static balancing conditions}

The expression of the partial derivative of the total potential energy $V$ of a mechanism with respect to the $n$-th generalized coordinate $\hat{q}_{n}$ typically assumes the following form $[1,2,8]$ :

$$
\frac{\partial V}{\partial \hat{q}_{n}}=\sum_{\psi} \Gamma_{n, \psi}(\mathbf{x}) \cdot \Xi_{n, \psi}(\hat{\mathbf{q}}) ; \quad \psi=1, . ., \Psi_{n} ; \quad n=1, . ., N
$$

where $\Gamma_{n, \psi}(\mathbf{x})$ is a constant coefficient (function of the unknown vector $\mathbf{x}$, i.e. of the parameters of the balancing elements) and $\Xi_{n, \psi}(\hat{\mathbf{q}})$ is a variable factor (function of the independent generalized coordinates).

Gravity compensation is achieved if Eq. (10) holds for any assumable configuration of the mechanism. Hence, a sufficient condition for static balancing is that all the constant coefficients of Eq. (A3) are null, i.e.:

$$
\Gamma_{n, \psi}(\mathbf{x})=0 ; \quad \psi=1, . ., \Psi_{n} ; \quad n=1, . ., N
$$

If for the examined mechanism variant VARe a solution to Eq. (A4) exists, exact gravity compensation can be granted. The solution of Eq. (A4) itself is a set of relations between the unknowns. The maximum number of relations obtained by imposing and solving Eq. (A4) is

$$
\sum_{n=1}^{N} \Psi_{n}
$$

However, a lower number of expression is generally obtained, since the same coefficient can be present in different partial derivatives.

The proposed algorithm starts from symbolic kinematic relations to write the expression of the mechanism potential energy $V$. Thank to this formulation, the exact partial derivatives of $V$ can be computed, hence permitting to find exact balancing solutions. In practice, the constant coefficients

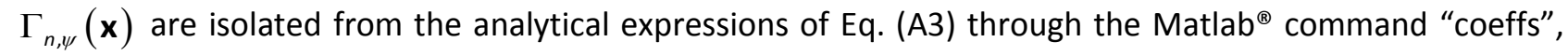
and the condition given by Eq. (A4) is imposed. A solution is determined by solving Eq. (A4) in the unknowns $m_{i}$ and $K_{\text {is }}$ (Matlab ${ }^{\circledR}$ command "solve").

It is worth noting that, except for Proc-2 and Proc-3, which exploit the symbolic solver, all the other operations performed within the algorithm are numerically computed. 


\section{A.3. Generation of mechanism variants with balancing elements}

For a generic mechanism with $L$ moving parts to which balancing elements can be attached, the number of variants with one counterweight, $E_{c w}$, is given by

$$
E_{c w}=L
$$

whereas, the number of variants with one spring, $E_{\text {spr, }}$ is given by all the possible combinations of $L+1$ members (since a spring can be attached to the fixed base as well) taken two at a time (since a spring connects two members):

$$
E_{s p r}=\frac{(L+1) !}{[(L+1)-2] ! \cdot 2 !}
$$

Hence, the total number of mechanism variants with a number of balancing element, $d$, that satisfies the condition $d \leq D$ max is given by all the possible combinations of $\left(E_{c w}+E_{s p r}\right)$ variants taken $d$ at a time:

$$
E_{\text {tot }}=\sum_{d=1}^{D_{\max }} \frac{\left(E_{c w}+E_{s p r}\right) !}{\left[\left(E_{c w}+E_{s p r}\right)-\mathrm{d}\right] ! \cdot d !}
$$

It should be noted that, since each variant does not admit repetitions (i.e. a single counterweight can be attached to each member, and a single spring can connect each couple of members), the maximum value of $D_{\text {max }}$ is equal to $\left(E_{c w}+E_{s p r}\right)$.

Equation (A6) clearly shows that the total number of mechanism variants to be generated and evaluated can grow quite fast with an increment in $D_{\max }$. In particular, under certain conditions, raising $D_{\max }$ by one may determine an increment of even one order of magnitude in $E_{\text {tot }}$, thus significantly increasing the computational time. Indeed, the execution of Proc- 2 and Proc-3 has been proven quite demanding. Nonetheless, the computational time of the proposed algorithm is deemed acceptable.

As for the case study presented in Section 3, by setting $D_{\max }=2, L=7$ and by excluding the fixed base from the possible spring connections, a total amount of 406 mechanism variant was generated and checked. In case $D_{\max }$ was set to 3 , a total of 3682 variants would be generated.

\section{References}

[1] C.M. Gosselin, J. Wang, Static balancing of spatial six degree-of-freedom parallel mechanisms with revolute actuators, J. Robotic Syst. 17 (3) (2000) 159-170.

[2] J. Wang, C.M. Gosselin, Static balancing of spatial four-degree-of-freedom parallel mechanisms, Mech. Mach. Theory 35 (4) (2000) 563-592.

[3] I. Simionescu, L. Ciupitu, The static balancing of the industrial robot arms: part I: discrete balancing, Mech. Mach. Theory 35 (2000) 1287-1298.

[4] I. Simionescu, L. Ciupitu, The static balancing of the industrial robot arms: part II: Continuous balancing, Mech. Mach. Theory 35 (2000) 1299-1311.

[5] I. Ebert-Uphoff, C.M. Gosselin, T. Laliberté, Static balancing of spatial parallel platform mechanismsrevisited, ASME J. Mech. Des. 122 (1) (2000) 43-51.

[6] I. Ebert-Uphoff, K. Johnson, Practical Considerations for the Use of Static Balancing for Parallel Kinematic Machines, Proc. IMechE Part K: J. Multi-body Dyn. 216 (1) (2002) 73-85.

[7] S.K. Agrawal, A. Fattah, Gravity-balancing of spatial robotic manipulators, Mech. Mach. Theory 39 (12) (2004) 1331-1344.

[8] M. Carricato, C.M. Gosselin, A Statically Balanced Gough/Stewart-Type Platform: Conception, Design, and Simulation, ASME J. Mech. Robot. 1 (3):031005 (2009) 1-16. 
[9] A. Martini, M. Troncossi, M. Carricato, A. Rivola, Elastodynamic behaviour of balanced closed-loop mechanisms: numerical analysis of a four-bar linkage, Meccanica 49 (3) (2014) 601-614.

[10] A. Martini, Gravity compensation of a 6-UPS parallel kinematics machine tool through elastically balanced constant-force generators, FME Transactions 46 (1) (2018) 10-16.

[11] S. Segla, C.M. Kalter-Kalkman, A.L. Schwab, Statical balancing of a robot mechanism with the aid of a genetic algorithm, Mech. Mach. Theory 33 (1/2) (1998) 163-174.

[12] C. Baradat, V. Arakelian, S. Briot, S. Guegan, Design and prototyping of a new balancing mechanism for spatial parallel manipulators, ASME J. Mech. Des. 130 (7):072305 (2008) 1-13.

[13] I. Simionescu, L. Ciupitu, L.C. Ionita, Static balancing with elastic systems of DELTA parallel robots, Mech. Mach. Theory 87 (2015) 150-162.

[14] A. Martini, M. Troncossi, M. Carricato, A. Rivola, Static balancing of a parallel kinematics machine with Linear-Delta architecture: theory, design and numerical investigation, Mech. Mach. Theory 90 (2015) 128-141.

[15] A. Martini, Development of an elastically compensated UPS-type constant-force generator for the static balancing of spatial parallel mechanisms. Proceedings of the XXIII AIMETA Conference, September 4-7, Salerno, Italy, pp. 241-249, 2017.

[16] T. Okada, H. Uchida, N. Uemura, Spring balancer apparatus, Patent EP0947296A2, 1999.

[17] K.-E. Forslund, Industrial robot, Patent US005901613A, 1999.

[18] A. Bayer, G. Merk, Industrial robot with a weight counterbalance system, Patent US20110072930A1, 2011.

[19] A. Broberg, R. Erkki, Industrial robot with pressurized air supply in balancing device, Patent US9221182B2, 2015.

[20] T. van Dam, P. Lambert, J. L. Herder, Static balancing of translational parallel mechanisms, Proceedings of the ASME 2011 International Design Engineering Technical Conferences \& Computers and Information in Engineering Conference IDETC/CIE 2011, August 28-31, 2011, Washington, USA.

[21] T. Wongratanaphisan, M. Chew, Gravity Compensation of Spatial Two-DOF Serial Manipulators, J. Robotic Syst. 19 (7) (2002) 329-347.

[22] A. Fattah, S. K. Agrawal, Gravity-Balancing of Classes of Industrial Robots, Proceedings of the 2006 IEEE International Conference on Robotics and Automation, Orlando, Florida - May 2006, pp. 28722877.

[23] M.J. Walker, R.S. Haines, An experimental study of the effects of counterweights on a six-bar chain, Mech. Mach. Theory 17 (6) (1982) 355-360.

[24] E. Raghu, A. Balasubramonian, Experimental study on the elastodynamic behavior of the unbalanced and the counterweighted four bar mechanisms, ASME J. Mech. Des. 112 (3) (1990) 271-277.

[25] A. Martini, M. Troncossi, A. Rivola, Elastodynamic effects of mass-balancing: experimental investigation of a four-bar linkage, Adv. Mech. Eng. DOI: 10.1155/2013/949457 (2013) 1-10.

[26] P.-Y. Lin, Design of statically balanced spatial mechanisms with spring suspensions, ASME J. Mech. Robot. 4 (2):021015 (2012) 1-7.

[27] Y.-Y. Lee, D.-Z. Chen, Determination of spring installation configuration on statically balanced planar articulated manipulators, Mech. Mach. Theory 74 (2014) 319-336.

[28] W.-H. Chiang, D.-Z. Chen, Design of planar variable-payload balanced articulated manipulators with actuated linear ground-adjacent adjustment, Mech. Mach. Theory 109 (2017) 296-312.

[29] L. Ciupitu, Adaptive Balancing of Robots and Mechatronic Systems, Robotics, 7 (4):68 (2018) 1-12. 
[30] R. Carrabotta, A. Martini, M. Troncossi, A. Rivola, Optimal static balancing of a spatial palletizing robot. Proceedings of the ECCOMAS Thematic Conference on Multibody Dynamics 2015, June 29 July 2, Barcelona, Spain, pp. 817-827, 2015.

[31] R.H. Nathan, A constant force generating mechanism, J Mech. Trans. Autom. Des. 107 (4) (1985) 508-512.

[32] L. Ciupitu, I. Simionescu, Zero-Free-Length Elastic Systems for Static Balancing, in: B. Corves, E.C. Lovasz, M. Hüsing, I. Maniu, C. Gruescu (Eds.), New Advances in Mechanisms, Mechanical Transmissions and Robotics - Mechanisms and Machine Science Vol. 46, Springer, Cham, 2017, pp. 59-67.

[33] J.L. Herder, On Static Balancing in Parallel Mechanisms. Proceedings of the Workshop on Fundamental Issues and Future Research Directions for Parallel Mechanisms and Manipulators, 3-4 October, Quebec City, Quebec, Canada, 2002.

[34] K. Deb, Optimization for Engineering Design: Algorithm and Examples. Prentice Hall, New Jersey, 1995.

[35] R.H. Byrd, J.C. Gilbert, J. Nocedal, A Trust Region Method Based on Interior Point Techniques for Nonlinear Programming, Math. Program. 89 (1) (2000) 149-185.

[36] V. Arakelian, The history of the creation and development of hand-operated balanced manipulators (HOBM), International Symposium on History of Machines and Mechanisms (Proceedings HMM 2004), pp. 347-356, Springer, Netherlands, 2004.

[37] V. Arakelian, S. Briot, Balancing of Linkages and Robot Manipulators, Mechanisms and Machine Science 27, Springer International Publishing, Switzerland, 2015.

[38] L. Sciavicco, B. Siciliano, Modelling and Control of Robot Manipulators, 2nd ed., Advanced Textbooks in Control and Signal Processing Series, Springer-Verlag, London, UK, 2000.

[39] A.A. Shabana, Dynamics of Multibody Systems, 3rd ed., Cambridge University Press, New York, USA 2005. 